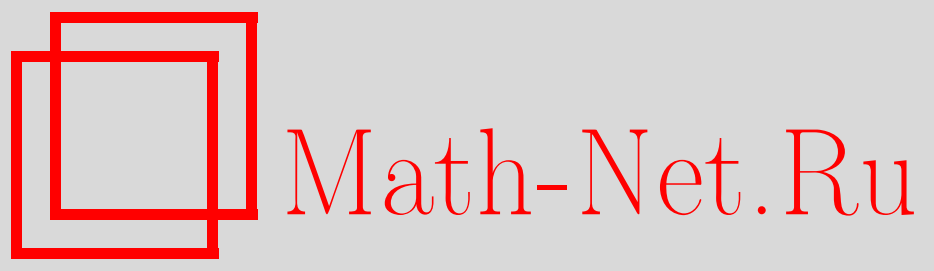

А. И. Оксак, Квантование стационарных гауссовских случайных процессов и их обобщений, ТМФ, 2012, том 173, номер 3, 479-516

DOI: https://doi.org/10.4213/tmf8341

Использование Общероссийского математического портала Math-Net.Ru подразумевает, что вы прочитали и согласны с пользовательским соглашением http://www.mathnet.ru/rus/agreement

Параметры загрузки:

IP : 34.239 .49 .27

26 апреля 2023 г., 17:37:02

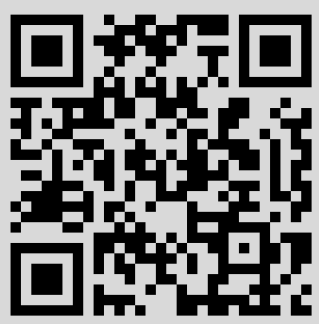




\title{
КВАНТОВАНИЕ СТАЦИОНАРНЫХ ГАУССОВСКИХ СЛУЧАЙНЫХ ПРОЦЕССОВ И ИХ ОБОБЩЕНИЙ
}

\begin{abstract}
Рассматривается квантование стационарных гауссовских случайных процессов, которым в физическом контексте соответствуют состояния открытых систем в равновесии с окружением. С этой целью предлагаются формализм и его физическая интерпретация в согласии с концепцией гамильтонова моделирования. Метод универсален и включает известные модели в качестве частных случаев. Рассмотрено расширение области применимости метода к линейным системам с инфракрасными особенностями двухточечных функций. В частности, фрактальные броуновские движения образуют семейство эталонных моделей этого класса.
\end{abstract}

Ключевые слова: открытые системы, гамильтоново моделирование, гауссовские потоки, квазисвободные состояния алгебры канонических коммутационных соотношений, фрактальные броуновские движения.

\section{1. ВВЕДЕНИЕ}

1.1. Концепция гамильтонова моделирования. Для квантования случайных процессов важен физический контекст. В физике случайные процессы служат средством описания поведения открытых систем. Под такими системами подразумевают ограниченную систему, взаимодействующую с окружением, в идеале с бесконечной средой. Роль среды заключается, в частности, в поддержании постоянной температуры. Среду называют термостатом, а открытую систему вместе с термостатом - полной системой. Согласно существующим физическим представлениям квантовое описание является наиболее точным, оно включает величину $\hbar$ (приведенную постоянную Планка). Классическое описание процесса является упрощением, имеющим дело с высокотемпературной асимптотикой. Квантованием случайного процесса называется его экстраполяция из классической области в квантовую. Однако единообразная процедура квантования возможна лишь для отдельных классов процессов, к которым относится рассмотренный в разделе 2 класс стационарных гауссовских случайных процессов с нулевым средним.

Как и для любого квантования, последовательные правила квантования случайных процессов должны выводиться из канонического формализма. В работе [1] был

* Российский заочный институт текстильной и легкой промышленности, Москва, Россия. E-mail: textleg@ruszitlp.ru, oksak.a@mail.ru 
впервые построен явный пример моделирования диссипативного случайного процесса в рамках гамильтонова формализма. До этого явные гамильтоновы реализации, например броуновского движения, отсутствовали. Поэтому результат работы [1] носит принципиальный характер (и не тривиален в свете дискуссий о “стреле времени").

Работа [1] породила целое направление - так называемое гамильтоново моделирование случайных процессов. Его концепция включает несколько положений.

1. Постулируется, что данный (классический) случайный процесс описывает поведение открытой системы. Для определенности рассматривается стационарный процесс.

2. Далее строится полная система (как правило, с бесконечным числом степеней свободы), являющаяся классической гамильтоновой системой с одновременными скобками Пуассона и эволюционирующая согласно детерминистическим уравнениям.

3. Полная система пребывает в состоянии теплового равновесия, а именно в гиббсовском состоянии. Случайные величины, описывающие открытую систему, составляют часть совокупности динамических переменных полной системы (поэтому в дальнейшем эти случайные величины также называются динамическими переменными).

4. Производя сужение гиббсовского состояния полной системы на подалгебру динамических переменных открытой системы, получают стационарное состояние открытой системы, которое должно совпасть с исходным процессом (вкратце это можно резюмировать так: в гамильтоновом моделировании ставится задача "погружения" данного случайного процесса в детерминированный и, более того, гамильтонов процесс).

Выполненное таким образом гамильтоново моделирование позволяет перейти к этапу квантования. Для полной системы он производится по общим правилам. Тепловому равновесию по-прежнему соответствует гиббсовское состояние полной системы. Производя сужение состояния на подалгебру динамических переменных открытой системы, получаем квантовый случайный процесс, экстраполирующий в квантовую область исходный классический случайный процесс.

В работах [2]-[7] было показано, как подобрать гамильтоновы модели для конкретных классических диссипативных процессов, эволюционирующих согласно стохастическим дифференциальным уравнениям (ланжевеновского типа). В работе [8] метод был распространен на пассивные системы, описываемые уравнениями ланжевеновского типа с запаздыванием. В работах данного направления основное внимание уделяется подбору термостата, поэтому мы назовем этот метод конструктивным ${ }^{1)}$. Специфика метода состоит в том, что динамические переменные открытой системы в фиксированный момент времени дополняются переменными термостата (своеобразными не-фон-неймановскими "скрытыми параметрами") так, чтобы образовалась полная система, удовлетворяющая требованиям 1-4. Достоинством подхода является то, что в нем не предполагается линейность модели. Однако “штучный" характер построения полной системы путем дополнения переменных открытой системы в фиксированный момент времени искусственными динамическими переменными термостата (не представляющими самостоятельного интереса) сужает область

\footnotetext{
1) Показательно название статьи [6] этого направления: "How to make a heat bath".
} 
применимости конструктивного подхода. Приходится использовать детальную процедуру подбора взаимодействия открытой системы с термостатом и начального состояния полной системы (см. [3], а также обзор [9]). “Технической” трудностью является то, что фиксация времени в переменных открытой системы не всегда возмож$\mathrm{Ha}^{2)}$, и тогда для преодоления ультрафиолетовых расходимостей требуется дополнительная регуляризация взаимодействия. Тем не менее для нелинейных моделей эти этапы конструктивного подхода к гамильтонову моделированию, по-видимому, являются необходимыми ${ }^{3)}$.

1.2. Гамильтоново моделирование в методе гауссовских потоков. Для линейных систем ситуация иная. Обобщая упомянутые выше конкретные модели, настоящая работа предлагает общий формализм гамильтонова моделирования стационарных гауссовских процессов, свободный от каких-либо трудностей. Мы назовем эту схему методом гауссовских потоков (по аналогии с потоками Гаусса в эргодической теории; [13], гл. 8, §2). Концепция гамильтонова моделирования укладывается в простую математическую схему с прозрачным физическим содержанием. Все известные линейные модели конструктивного подхода входят в данную схему как частные случаи.

Трудности конструктивного метода преодолеваются в настоящей работе отказом от моделирования термостата. Вместо этого предлагается универсальная конструкция "минимальной" полной системы для данной открытой системы. Помимо перечисленных выше общих требований 1-4 она характеризуется тем, что алгебра динамических переменных полной системы строится из динамических переменных открытой системы, взятых за времена от $-\infty$ до $+\infty$. В методе гауссовских потоков явное выделение переменных термостата из полной системы не производится, и, таким образом, этап моделирования переменных термостата отсутствует. Это не означает отсутствие термостата. Разумеется, исключение составляет случай, когда открытая система является гамильтоновой с детерминированной эволюцией (в классическом режиме); тогда она сама по себе может считаться "минимальной" полной системой. Если же характер эволюции открытой системы (в классическом режиме) недетерминированный, то это означает появление с течением времени новых степеней свободы в открытой системе, что указывает на присутствие термостата.

Результатом гамильтонова моделирования является превращение открытой системы в систему канонического формализма, но с двухвременными скобками Пуассона и, соответственно, с двухвременными коммутационными соотношениями для динамических переменных открытой системы на классическом и квантовом уровнях. Что касается полной системы, то ее динамика определяется одновременными скобками Пуассона и гамильтонианом.

В разделе 2 показано, как применяется для квантования метод гауссовских потоков ко всем стационарным гауссовским случайным процессам с нулевым средним (это ответ на вопрос о существовании). Соответствующие квантовые стационарные

\footnotetext{
2) Хорошо известным примером такого рода является скорость классического эйнштейновского броуновского движения (белый шум), которая в фиксированный момент времени не существует как случайная величина.

3) Применению аппарата функционального интегрирования к данной проблеме посвящены работы [10]-[12].
} 
гауссовские случайные процессы однозначно определяются классическими двухточечными корреляционными функциями динамических переменных открытой системы. Как следствие, явные формулы квантования (4), (7) дают ответ на вопрос о единственности, в том смысле, что квантовая экстраполяция случайного процесса приводит к однозначному результату. Разумеется, рассматриваемый метод квантования применим и тогда, когда построение явной модели в конструктивном методе проблематично, например как в случае осцилляторного броуновского процесса по Эйнштейну или в случае разветвленной электрической цепи с резисторами, конденсаторами, индуктивностями.

В разделе 3 рассмотрено дальнейшее расширение области применимости метода гауссовских потоков к случаю некоторых систем с инфракрасными особенностями корреляционных функций и с ненормируемым стационарным обобщенным состоянием. Эта схема описывает поведение открытой системы с конечным числом степеней свободы, погруженной в термодинамически равновесную среду с бесконечным числом степеней свободы. При некоторых группах симметрий открытой системы полная система не обладает стационарным состоянием. Тем не менее благодаря квазилокальному характеру взаимодействия открытой системы с термостатом процессы полной системы протекают на фоне равновесного состояния среды при определенной температуре. В этот класс попадают, например, процессы свободного броуновского движения по Эйнштейну и по Ланжевену, фрактальные броуновские движения (в конструктивном подходе гамильтоново моделирование проделано только для свободного броуновского движения по Ланжевену [14]).

1.3. Основные формулы метода гауссовских потоков. Для описания многокомпонентного гауссовского случайного процесса мы используем векторный случайный процесс $X(t)$ над $L$. Это линейный оператор, зависящий от температуры как от параметра и от времени $t$, который сопоставляет векторам $l$ из некоторого вещественного линейного пространства $L$ вещественные (в квантовом случае эрмитовы) случайные величины $X(t) l$. Компоненты вектора $X(t)$ интерпретируются как динамические переменные некоторой открытой системы (например, как координата и импульс броуновской частицы в процессе Орнштейна-Уленбека). Классические и квантовые средние обозначаются соответственно как $\langle\cdot\rangle^{\mathrm{cl}}$ и $\langle\cdot\rangle^{\mathrm{qu}}$. Они определяют состояние алгебры динамических переменных. Поскольку ставится задача квантования классического процесса, то он предполагается заданным; квантовый процесс должен быть построен. По условию классический процесс является стационарным гауссовским с нулевым средним:

$$
\langle X(t) l\rangle^{\mathrm{cl}}=0, \quad l \in L .
$$

Считаются заданными классические парные корреляционные функции, имеющие следующую структуру:

$$
\left\langle X(s) l_{1} \cdot X(t) l_{2}\right\rangle^{\mathrm{cl}}=\beta^{-1} C\left(s-t ; l_{1}, l_{2}\right), \quad l_{1}, l_{2} \in L,
$$

где $\beta=1 / k T$ - температурный модуль $(k$ - постоянная Больцмана, $T>0$ - абсолютная температура). В физике равновесные корреляционные функции проистекают из гиббсовского распределения. Это служит мотивировкой появления параметра температуры в соотношении (2) и в дальнейших формулах. Величина $C\left(s-t ; l_{1}, l_{2}\right)-$ 
вещественная билинейная форма над $L$, зависящая от разности $s-t$, что означает стационарность процесса. Множитель $\beta^{-1}$ учитывает явную зависимость классических корреляционных функций от температуры. Однако мы допускаем возможность того, что классические корреляционные функции первоначально были заданы абстрактно математически (без какой-либо температурной зависимости). Тогда им следует приписать некоторую температуру $T_{0}$ и выражением

$$
\left\langle X(s) l_{1} \cdot X(t) l_{2}\right\rangle^{\mathrm{cl}}=\left.\left\langle X(s) l_{1} \cdot X(t) l_{2}\right\rangle^{\mathrm{cl}}\right|_{T=T_{0}} \cdot \frac{T}{T_{0}}
$$

доопределить процесс при любой температуре (как в (2)).

В разделе 2 изложены все этапы гамильтонова моделирования. Они включают единообразную конструкцию фазового пространства "минимальной" полной системы (п. 2.2), каноническое квантование полной системы (п. 2.3) и, как следствие, квантование исходной открытой системы (п. 2.4).

Результатом моделирования является выражение двухвременных скобок Пуассона динамических переменных открытой системы через классические корреляционные функции (2):

$$
\left\{X(s) l_{1}, X(t) l_{2}\right\}=-\beta \partial_{s}\left\langle X(s) l_{1} \cdot X(t) l_{2}\right\rangle^{\mathrm{cl}}, \quad l_{1}, l_{2} \in L,
$$

где $\partial_{s}$ - оператор дифференцирования по $s$. При квантовании скобки Пуассона заменяются коммутатором с множителем $i / \hbar$, так что

$$
\left[X(s) l_{1}, X(t) l_{2}\right]=\Lambda\left\langle X(s) l_{1} \cdot X(t) l_{2}\right\rangle^{\mathrm{cl}}, \quad l_{1}, l_{2} \in L,
$$

где

$$
\Lambda=i \beta \hbar \partial_{s}
$$

Мы приходим к следующему выводу: квантовая структура открытой системы полностью определяется классическими двухточечными корреляционными функциями только открытой системы.

Для двухточечной функции гауссовского вакуумного состояния квантовой открытой системы (при $T=0$ ) имеет место формула

$$
\left\langle X(s) l_{1} \cdot X(t) l_{2}\right\rangle_{0}^{\mathrm{qu}}=\Lambda_{+}\left\langle X(s) l_{1} \cdot X(t) l_{2}\right\rangle^{\mathrm{cl}}, \quad l_{1}, l_{2} \in L,
$$

где использована функция $x_{+}=x \cdot \theta(x), \theta(x)=(1+\operatorname{sgn} x) / 2$ (в силу $(2)$ правые части в (4) и (6) не зависят от $\beta$ ).

Гиббсовское (гауссовское) состояние квантовой открытой системы характеризуется своими квантовыми двухточечными корреляционными функциями, которые также выражаются через (2):

$$
\left\langle X(s) l_{1} \cdot X(t) l_{2}\right\rangle^{\mathrm{qu}}=\mathrm{B}(\Lambda)\left\langle X(s) l_{1} \cdot X(t) l_{2}\right\rangle^{\mathrm{cl}}, \quad l_{1}, l_{2} \in L,
$$

где

$$
\mathrm{B}(x)=\frac{x}{2}\left(\operatorname{ctg} \frac{x}{2}+1\right) .
$$

Квантовое среднее от векторного случайного процесса равно нулю, как и в классическом случае:

$$
\langle X(t) l\rangle^{\mathrm{qu}}=0, \quad l \in L .
$$


Отметим, что при $T=0$ двухточечная функция (7) переходит в (6), а при $T \rightarrow \infty$ после симметризации относительно перестановки $\left(s, l_{1}\right) \leftrightarrow\left(t, l_{2}\right)$ переходит в $(2)$. Таким образом, формулы для классического состояния и для квантового вакуумного состояния получаются из квантового температурного состояния в двух предельных режимах: при $T \rightarrow \infty$ и при $T=0$.

1.4. Замечание о временных флуктуациях в статистической термодинамике. Существуют два конкурирующих подхода к вычислениям термодинамических флуктуаций - на основе статистической механики Гиббса и на основе статистической термодинамики Эйнштейна. При этом физические результаты двух подходов не всегда совпадают (см. обзор [15]). В книге [16] случайные процессы, названные временными флуктуациями, излагаются с позиций статистической термодинамики Эйнштейна. Сравнение формул (122.9) и (124.18) из [16] дает важное правило перехода от классической двухточечной корреляционной функции в фурье-представлении к квантовой:

$$
\left(x^{2}\right)_{\omega} \rightarrow\left(x^{2}\right)_{\omega} \frac{\hbar \omega}{2 T} \operatorname{cth} \frac{\hbar \omega}{2 T} .
$$

Вывод этого правила использует аппарат, связывающий двухточечную корреляционную функцию с обобщенной восприимчивостью, и флуктуационно-дисперсионную теорему. При этом возможность гамильтоновой реализации моделей подразумевается как данность (которая не очевидна). В отличие от [16], целью раздела 2 настоящей работы является не только вывод формул (4)-(7), но и доказательство возможности последовательной процедуры гамильтонова моделирования некоторого класса случайных процессов. Формулы (4)-(7) позволяют осуществить квантование процесса, если известны классические двухвременные корреляционные функции. В частности, симметризованный вариант формулы (7) является аналогом (10). Всюду, как и в [16], условие гауссовости процессов играет решающую роль.

1.5. Список обозначений. Приведем для удобства список обозначений, используемых в статье.

$\mathcal{A}^{\mathrm{cl}}(\chi), \mathcal{A}^{\mathrm{qu}}(\chi), \mathcal{A}^{\sharp}(\chi)-*$-алгебра полной системы (п. 3.8);

$\mathcal{A}^{\mathrm{cl}}\left(\chi_{0}\right), \mathcal{A}^{\mathrm{qu}}\left(\chi_{0}\right), \mathcal{A}^{\sharp}\left(\chi_{0}\right)-$ *-подалгебра (п. 3.8);

$b(z)$ - вектор из $H_{0}^{\text {qu }}(143)$;

$\mathrm{B}(x)$ - функция (8);

$\langle\ldots\rangle^{\mathrm{cl}},\langle\ldots\rangle^{\mathrm{qu}}-$ среднее или виртуальное среднее (классическое или квантовое) (п. 1.3, 3.1);

$\mathcal{E}_{u}$ - унитарные элементы (110);

$\tilde{f}(\lambda) \equiv F_{t \rightarrow \lambda}[f(t)]$ - преобразование Фурье $(24) ;$

$|\mathrm{GIBBS}\rangle^{\mathrm{cl}},|\mathrm{GIBBS}\rangle^{\mathrm{qu}},|\mathrm{GIBBS}\rangle^{\sharp}$ - циклический вектор (п. 3.9);

$H^{\mathrm{cl}}-$ пополнение $\chi$ (п. 2.1, 3.6);

$H_{0}^{\mathrm{cl}}, H_{0}^{\mathrm{qu}}-$ пополнение $\chi_{0}$ (п. 3.6, 3.9);

Н - гамильтониан полной системы (30), (104);

$\mathcal{H}^{\mathrm{cl}}, \mathcal{H}^{\mathrm{qu}}, \mathcal{H}^{\sharp}-$ пространство представления *-алгебры (п. 3.10);

$\mathcal{H}_{0}^{\mathrm{cl}}, \mathcal{H}_{0}^{\mathrm{qu}}, \mathcal{H}_{0}^{\sharp}-$ пространство представления $*$-подалгебры (п. 3.9); 
$\mathcal{J}^{\mathrm{cl}}, \mathcal{J}^{\text {qu }}, \mathcal{J}^{\sharp}-$ *-идеал (п. 3.8);

$J$ - оператор комплексной структуры (22), (23), (54);

$L$ - фиксированное вещественное векторное пространство (п. 2.1, 3.2);

$m^{\mathrm{cl}}, m^{\mathrm{qu}}, m^{\sharp}-$ обобщенное условное ожидание (п. 3.8);

$P: \mathcal{Z} \rightarrow \chi-$ сечение расслоения (п. 3.11);

$\mathbb{R}$ - вещественная прямая;

$s^{\mathrm{cl}}, s^{\mathrm{qu}}, s^{\sharp}-$ обобщенное состояние $*$-алгебры (127);

$s_{0}^{\mathrm{cl}}, s_{0}^{\mathrm{qu}}, s_{0}^{\sharp}-$ гауссовское состояние *-подалгебры (118), (120);

$S(\mathbb{R})$ и $S_{\mathrm{r}}(\mathbb{R})$ - комплексное и вещественное пространства Шварца основных функций;

$\mathbf{S}=S_{\mathrm{r}}(\mathbb{R}) \otimes L($ п. 2.1, 3.3);

$(u \mid \nu)^{\mathrm{cl}}-$ скалярное произведение (14), (74);

$\|u\|^{\mathrm{cl}},\|u\|^{\text {qu }}$ - нормы (84), (122);

$U(\tau)$ - оператор (18), (75);

$\chi=\mathbf{S} / \mathbf{S}^{\perp}-$ фазовое пространство полной системы (п. 2.1, 3.3);

$\chi_{0}$ - линеал в $\chi$ (п. 3.8);

$X(t)$ - векторный обобщенный случайный или виртуальный процесс (п. $2.1,3.2)$;

$X[\Phi]$ - динамические переменные открытой системы (п. 2.1, 3.3);

$Y^{\mathrm{cl}}(0)$ - динамические переменные классической полной системы (27), (103);

$Y^{\mathrm{qu}}(0)$ - динамические переменные квантовой полной системы $(41),(103)$;

$\mathcal{Z}=\chi / \chi_{0}-$ пространство зарядов (п. 3.5);

$Z: \chi \rightarrow \mathcal{Z}$ - проекция расслоения (п. 3.5);

$\mathbf{Z}$ - оператор заряда (134);

$\gamma_{g}$ - калибровочное преобразование (114);

$\pi^{\mathrm{cl}}, \pi^{\mathrm{qu}}, \pi^{\sharp}$ - температурное представление $*$-алгебры (п. 3.10);

$\pi_{0}^{\mathrm{cl}}, \pi_{0}^{\mathrm{qu}}, \pi_{0}^{\sharp}-$ гиббсовское представление $*$-подалгебры (п. 3.9);

$\sigma(u, \nu)$ - симплектическая форма (29), (102);

$\omega$ - оператор в $H_{0}^{\text {qu }}(123)$;

$\Omega$ - оператор в $H^{\mathrm{cl}}$ или в $H_{0}^{\mathrm{cl}}((23)$ или $(95))$.

\section{2. ГАМИЛЬТОНОВЫ МОДЕЛИ СТАЦИОНАРНЫХ ГАУССОВСКИХ СЛУЧАЙНЫХ ПРОЦЕССОВ}

2.1. Свойства двухточечной корреляционной функции. Здесь мы покажем, что формулы квантования (4), (7) для стационарных гауссовских случайных процессов с нулевым средним являются результатом гамильтонова моделирования. Отправной точкой служит векторный случайный процесс $X(t)$ над $L$. Он задается средними (1), (2). Случайные величины $X(t) l$ интерпретируются как переменные, описывающие эволюцию некоторой классической открытой системы. Задача состо- 
ит в том, чтобы построить гамильтонову модель соответствующей полной системы и гиббсовские состояния ее квантового аналога.

Чтобы включить в рассмотрение такие процессы, как белый шум, мы рассматриваем $X(t)$ как векторный обобщенный случайный процесс. Сглаживание производится с вещественными основными функциями $\varphi$ от вещественной переменной $t$ из (вещественного) пространства Шварца $S_{\mathrm{r}}(\mathbb{R})$. Это значит, что каждому вектору $l$ из $L$ и основной функции $\varphi$ из $S_{\mathrm{r}}(\mathbb{R})$ сопоставляется билинейным образом вещественная случайная величина $\int X(t) l \cdot \varphi(t) d t$ из некоторого линейного пространства вещественных гауссовских случайных величин с нулевым средним. Для удобства примем, что размерность этих величин в физических единицах равна нулю. В соответствии с (1), (2) имеем выражения для среднего

$$
\left\langle\int X(t) l \cdot \varphi(t) d t\right\rangle^{\mathrm{cl}}=0
$$

и для ковариации

$$
\beta^{-1} \cdot \int C\left(s-t ; l_{1}, l_{2}\right) \varphi(s) \psi(t) d s d t
$$

случайных величин

$$
\int X(s) l_{1} \cdot \varphi(s) d s, \quad \int X(t) l_{2} \cdot \psi(t) d t
$$

которые получаются сглаживанием (2) по $s, t$ с $\varphi(s) \psi(t)$. Корреляционная функция (2) есть вещественная обобщенная функция умеренного роста по $s-t$. При этом выполняется естественное требование непрерывности по $\varphi$ :

$$
\int X(t) l \cdot \varphi_{n}(t) d t \rightarrow 0 \quad \text { в среднеквадратичном, если } \quad \varphi_{n} \rightarrow 0 \quad \text { в } \quad S_{\mathrm{r}}(\mathbb{R}) .
$$

Благодаря билинейности относительно $\varphi$ и $l$ случайную величину $\int X(t) l \cdot \varphi(t) d t$ можно записать в виде $X[\varphi \otimes l]$, где $X$ есть линейный оператор над вещественным векторным пространством - алгебраическим тензорным произведением ${ }^{4)} \mathbf{S}:=$ $S_{\mathrm{r}}(\mathbb{R}) \otimes L$. Он сопоставляет каждому вектору $\Phi \in \mathbf{S}$ вида $\Phi:=\sum_{j=1}^{N} \varphi_{j} \otimes l_{j}$ гауссовскую случайную величину $X[\Phi]:=\sum_{j=1}^{N} X\left[\varphi_{j} \otimes l_{j}\right]$.

Имеют место соотношения

$$
\begin{array}{rlrl}
\langle X[\Phi]\rangle^{\mathrm{cl}} & =0, & & \Phi \in \mathbf{S}, \\
\beta\left\langle X[\Phi]^{2}\right\rangle^{\mathrm{cl}} & \geqslant 0, & \Phi \in \mathbf{S},
\end{array}
$$

причем левая часть (12) обращается в нуль, только если $X[\Phi]=0$. Введем линеал (т. е. линейное подпространство)

$$
\mathbf{S}^{\perp}=\left\{\Phi \in \mathbf{S}: \beta \cdot\left\langle X[\Phi]^{2}\right\rangle^{\mathrm{cl}}=0\right\} .
$$

Факторпространство $\chi:=\mathbf{S} / \mathbf{S}^{\perp}$ с естественной проекцией $x: \mathbf{S} \rightarrow \chi$ есть предгильбертово пространство со скалярным произведением $(u \mid \nu)^{\text {cl }}$ векторов $u:=x[\Phi]$, $\nu:=x[\Psi]:$

$$
(u \mid \nu)^{\mathrm{cl}} \equiv(x[\Phi] \mid x[\Psi])^{\mathrm{cl}}:=\beta \cdot\langle X[\Phi] \cdot X[\Psi]\rangle^{\mathrm{cl}}, \quad \Phi, \Psi \in \mathbf{S} .
$$

\footnotetext{
4) Символ := означает равенство по определению.
} 
Вещественное гильбертово пространство $H^{\mathrm{cl}}$ со скалярным произведением $(u \mid \nu)^{\mathrm{cl}}$ определяется как пополнение $\chi$.

Имея в виду дальнейшую процедуру квантования (п. 2.3, 2.4), мы различаем вектор $x[\Phi]$ из $H^{\text {cl }}$ и случайную величину $X[\Phi]$. По аналогии с формулой $\int X(t) l \times$ $\varphi(t) d t=X[\varphi \otimes l]$ формула $\int x(t) l \cdot \varphi(t) d t=x[\varphi \otimes l]$ вводит векторную обобщенную функцию $x(t)$.

При $\tau \in \mathbb{R}$ положим

$$
\varphi_{\tau}(t):=\varphi(t-\tau), \quad(\varphi \otimes l)_{\tau}:=\varphi_{\tau} \otimes l .
$$

Тогда на $\mathbf{S}$ определено линейное представление

$$
\Phi=\sum_{j} \varphi_{j} \otimes l_{j} \mapsto \Phi_{\tau}:=\sum_{j}\left(\varphi_{j} \otimes l_{j}\right)_{\tau}
$$

эволюционной группы $\mathbb{R}$ временных трансляций. Благодаря зависимости корреляционной функции (2) от $s, t$ через разность $s-t$ выполнено условие стационарности

$$
\beta \cdot\left\langle X\left[\Phi_{\tau}\right] \cdot x\left[\Psi_{\tau}\right]\right\rangle^{\mathrm{cl}}=\beta \cdot\langle X[\Phi] \cdot x[\Psi]\rangle^{\mathrm{cl}}, \quad \Phi, \Psi \in \mathbf{S}, \quad \tau \in \mathbb{R} .
$$

Тем самым в $H^{\mathrm{cl}}$ задано изометрическое представление $U(\tau)$ эволюционной группы $\mathbb{R}$, действующей на плотном линеале $\chi \subset H^{\mathrm{cl}}$ по правилу

$$
U(\tau) X[\Phi]:=X\left[\Phi_{\tau}\right], \quad \Phi \in \mathbf{S}, \quad \tau \in \mathbb{R} .
$$

В частности,

$$
U(\tau) x(t) l:=x(t+\tau) l, \quad l \in L, \quad t, \tau \in \mathbb{R} .
$$

Генератор представления

$$
D:=\left.\partial_{\tau} U(\tau)\right|_{\tau=0}
$$

есть кососимметрический оператор с областью определения, содержащей $\chi$.

В разделе 2, кроме замечания 1, мы не рассматриваем процессы с ненулевыми стационарными векторами в $H^{\mathrm{cl}}$, т. е. мы предполагаем, что

$$
\left\{u \in H^{\mathrm{cl}}: U(\tau) u=u \text { при всех } \tau \in \mathbb{R}\right\} \equiv D^{-1}\{0\}=\{0\} .
$$

Это условие, означающее отсутствие "нулевых мод" процесса, необходимо для дальнейшего построения гиббсовского состояния. В замечании 1 в п. 2.4 с помощью предельного перехода мы распространим результат на процессы, в которых условие (21) не выполнено.

В предположении (21) генератор $D$ есть произведение самосопряженного положительного оператора $\Omega:=\left(-D^{2}\right)^{1 / 2}$ и кососимметрического оператора $J:=D \Omega^{-1}$ такого, что

$$
\begin{array}{r}
J^{2}=-1, \\
D=\Omega J=J \Omega .
\end{array}
$$

Тогда оператор эволюции имеет вид

$$
U(\tau)=e^{D \tau}=e^{J \Omega \tau}=\cos (\Omega \tau)+J \sin (\Omega \tau)=\int(\cos (\lambda \tau)+J \sin (\lambda \tau)) d E(\lambda)
$$


Здесь и далее $d E(\lambda)$ и $d \widehat{E}(\lambda)$ - спектральные меры операторов $\Omega$ и $\widehat{\Omega}=-\Omega$. Определим преобразования Фурье функции или обобщенной функции $f(t)$, полагая

$$
\tilde{f}(\lambda) \equiv F_{t \rightarrow \lambda}[f(t)]:=\int e^{i \lambda t} f(t) d t
$$

Тогда

$$
F_{\tau \rightarrow \lambda}\left[(u \mid U(\tau) \nu)^{\mathrm{cl}}\right] \frac{d \lambda}{2 \pi}=\frac{1}{2}(u \mid(d E(\lambda)+d \widehat{E}(\lambda)) \nu)^{\mathrm{cl}}+\frac{i}{2}(u \mid(d E(\lambda)-d \widehat{E}(\lambda)) J \nu)^{\mathrm{cl}} .
$$

Следовательно, при любых $u, \nu \in H^{\mathrm{cl}}$

$$
F_{\tau \rightarrow \lambda}\left[(u \mid U(\tau) u)^{\mathrm{cl}}\right] \frac{d \lambda}{2 \pi} \in \mathrm{M}^{+}(\mathbb{R}), \quad F_{\tau \rightarrow \lambda}\left[(u \mid U(\tau) \nu)^{\mathrm{cl}}\right] \frac{d \lambda}{2 \pi} \in \mathrm{M}(\mathbb{R}),
$$

где $\mathrm{M}^{+}(\mathbb{R})$ и $\mathrm{M}(\mathbb{R})$ - множества соответственно неотрицательных и комплексных конечных борелевских мер на $\mathbb{R}$. В силу положительности $\Omega$

$$
\int_{\lambda=0} d E(\lambda)=0, \quad \int_{\lambda=0} d \widehat{E}(\lambda)=0,
$$

так что (25) можно усилить: при любых $u, \nu \in H^{\mathrm{cl}}$

$$
F_{\tau \rightarrow \lambda}\left[(u \mid U(\tau) u)^{\mathrm{cl}}\right] \frac{d \lambda}{2 \pi} \in \mathrm{M}_{0}^{+}(\mathbb{R}), \quad F_{\tau \rightarrow \lambda}\left[(u \mid U(\tau) \nu)^{\mathrm{cl}}\right] \frac{d \lambda}{2 \pi} \in \mathrm{M}_{0}(\mathbb{R}),
$$

где $\mathrm{M}_{0}^{+}(\mathbb{R})$ и $\mathrm{M}_{0}(\mathbb{R})$ - множества соответственно неотрицательных и комплексных конечных борелевских мер $d m(\lambda)$ на $\mathbb{R}$ с нулевым вкладом в точке $\lambda=0$, т.е. $\int_{\lambda=0} d m(\lambda)=0$. Заметим, что условие (25) имеет место и при наличии "нулевых мод", поэтому (26) есть альтернативная формулировка условия (21) непосредственно в терминах двухточечной функции.

2.2. Фазовое пространство “минимальной” полной системы. В методе гауссовских потоков эволюция полной системы описывается многокомпонентным случайным вектором $Y^{\mathrm{cl}}(t)$ над гильбертовым пространством $H^{\mathrm{cl}}$, который сопоставляет вектору $u \in H^{\mathrm{cl}}$ вещественную случайную величину $Y^{\mathrm{cl}}(t) u$, линейно зависящую от $u$. Мы покажем, что в качестве $Y^{\mathrm{cl}}(t)$ можно принять выражение

$$
Y^{\mathrm{cl}}(t)=Y^{\mathrm{cl}} U(t)
$$

где $\beta^{1 / 2} Y^{\mathrm{cl}}$ - векторная случайная величина над $H^{\mathrm{cl}}$ со стандартным гауссовским распределением. Имеем

$$
\left\langle Y^{\mathrm{cl}} u\right\rangle^{\mathrm{cl}}=0, \quad\left\langle Y^{\mathrm{cl}} u \cdot Y^{\mathrm{cl}} \nu\right\rangle^{\mathrm{cl}}=\beta^{-1}(u \mid \nu)^{\mathrm{cl}}, \quad u, \nu \in H^{\mathrm{cl}} .
$$

Канонический формализм применительно к полной системе выглядит следующим образом. Для одновременных скобок Пуассона динамических переменных $Y^{\mathrm{cl}}(t) u$ постулируется выражение

$$
\left\{Y^{\mathrm{cl}}(t) u, Y^{\mathrm{cl}}(t) \nu\right\} \equiv \sigma(u, \nu):=-(D u \mid \nu)^{\mathrm{cl}}=(u \mid D \nu)^{\mathrm{cl}}, \quad u, \nu \in \chi .
$$


Здесь $\chi$ является фазовым пространством полной системы с симплектической (т. е. невырожденной кососимметрической билинейной) формой $\sigma(u, \nu)$ и линейной эволюцией (18). В таком случае энергия вектора $u \in \chi$ определяется формулой

$$
E(u)=\left.\frac{1}{2} \partial_{\tau} \sigma\left(u_{\tau}, u\right)\right|_{\tau=0}=\frac{1}{2} \sigma(D u, u)=\frac{1}{2}(D u \mid D u)^{\mathrm{cl}} .
$$

$\mathrm{C}$ каждым вектором $u \in \chi$ ассоциируется микросостояние $\mathrm{s}_{u}$ с нулевой дисперсией элементов алгебры, порожденной $Y^{\mathrm{cl}}(t)$, а именно

$$
\mathrm{s}_{u}\left(Y^{\mathrm{cl}}(0) \nu\right):=\sigma(u, \nu)=-(D u \mid \nu)^{\mathrm{cl}} .
$$

Его энергия считается равной энергии вектора $u$ :

$$
E(u)=\frac{1}{2}(D u \mid D u)^{\mathrm{cl}}=\frac{1}{2} \sum_{a}\left[\left(D u \mid e_{a}\right)^{\mathrm{cl}}\right]^{2}=\mathrm{s}_{u}\left(\frac{1}{2} \sum_{a}\left(Y^{\mathrm{cl}}(0) e_{a}\right)^{2}\right),
$$

где $\left\{e_{a}\right\}$ - ортонормированный базис в $H^{\mathrm{cl}}$, символ $а$ пробегает некоторое индексное множество. Гамильтониан Н полной системы есть функционал от векторной динамической переменной $Y^{\mathrm{cl}}(t)$ (при фиксированном $t$ ). Он выбирается таким образом, что $E(u)=\mathrm{s}_{u}(\mathrm{H})$ при любом $u$, откуда находим

$$
\mathrm{H} \equiv \mathrm{H}\left(Y^{\mathrm{cl}}(t)\right):=\frac{1}{2} \sum_{a}\left(Y_{a}^{\mathrm{cl}}(t)\right)^{2},
$$

где $Y_{a}^{\mathrm{cl}}(t):=Y^{\mathrm{cl}}(t) e_{a}$. Решением гамильтоновых уравнений движения

$$
\begin{aligned}
\partial_{t} Y^{\mathrm{cl}}(t) u & =\left\{H, Y^{\mathrm{cl}}(t) u\right\}=\sum_{a} Y_{a}^{\mathrm{cl}}(t)\left\{Y^{\mathrm{cl}}(t) e_{a}, Y^{\mathrm{cl}}(t) u\right\}=\sum_{a} Y_{a}^{\mathrm{cl}}(t)\left(D u \mid e_{a}\right)^{\mathrm{cl}}= \\
& =Y^{\mathrm{cl}}(t) \sum_{a} e_{a}\left(D u \mid e_{a}\right)^{\mathrm{cl}}=Y^{\mathrm{cl}}(t) D u
\end{aligned}
$$

с начальным условием $\left.Y^{\mathrm{cl}}(t) u\right|_{t=0}=Y^{\mathrm{cl}}(0) u$, очевидно, является

$$
Y^{\mathrm{cl}}(t) u=Y^{\mathrm{cl}}(0) U(t) u \text {. }
$$

Вместе с (29) при $t=0$ это дает выражение для двухвременных скобок Пуассона

$$
\left\{Y^{\mathrm{cl}}(s) u, Y^{\mathrm{cl}}(t) \nu\right\}=(u \mid \mathcal{D}(s-t) \nu),
$$

где введена так называемая перестановочная функиия

$$
\mathcal{D}(s-t):=D U(t-s)=-\partial_{s} U(t-s) .
$$

Полная система пребывает в состоянии теплового равновесия, т. е. имеет каноническое распределение Гиббса с температурой $T$ (которое формально совпадает с большим каноническим распределением, если система состоит из частиц при нулевом химическом потенциале). В случае конечномерного $H^{\mathrm{cl}}\left(\operatorname{dim} H^{\mathrm{cl}}=2 N\right)$ характеристический функционал состояния представляет собой интеграл по множеству микросостояний $\left\{\mathrm{s}_{\nu}\right\}$. При $u \in H^{\mathrm{cl}}$ имеем

$$
\left\langle e^{i Y^{\mathrm{cl}}(0) u}\right\rangle^{\mathrm{cl}}=\frac{Q(u)}{Q},
$$


где

$$
Q(u):=\int \mathrm{s}_{\nu}\left(e^{i Y^{\mathrm{cl}}(0) \nu} \cdot e^{-\beta \mathrm{H}}\right) d^{2 N} \nu,\left.\quad Q \equiv Q(u)\right|_{u=0}
$$

В результате

$$
\left\langle e^{i Y^{\mathrm{cl}}(0) u}\right\rangle^{\mathrm{cl}}=\exp \left(-\sum_{a} \frac{\left(u_{a}\right)^{2}}{2 \beta}\right)=e^{-(u \mid u)^{\mathrm{cl}} / 2 \beta}
$$

с $u_{a}:=\left(u \mid e_{a}\right)^{\mathrm{cl}}$. Это значит, что компоненты $Y_{a}^{\mathrm{cl}}(0)$ являются независимыми гауссовскими случайными величинами с нулевым средним и с дисперсией $\beta^{-1}$ :

$$
\left\langle Y_{a}^{\mathrm{cl}}(0) \cdot Y_{b}^{\mathrm{cl}}(0)\right\rangle^{\mathrm{cl}}=\beta^{-1} \delta_{a b}, \quad\left\langle Y^{\mathrm{cl}}(0) u \cdot Y^{\mathrm{cl}}(0) \nu\right\rangle^{\mathrm{cl}}=\beta^{-1}(u \mid \nu)^{\mathrm{cl}},
$$

так что

$$
\begin{gathered}
\left\langle Y^{\mathrm{cl}}(t) u\right\rangle^{\mathrm{cl}}=0, \quad\left\langle Y^{\mathrm{cl}}(s) u \cdot Y^{\mathrm{cl}}(t) \nu\right\rangle^{\mathrm{cl}}=\left(u \mid W^{\mathrm{cl}}(s-t) \nu\right)^{\mathrm{cl}}, \quad u, \nu \in H^{\mathrm{cl}}, \\
W^{\mathrm{cl}}(s-t)=\beta^{-1} U(t-s) .
\end{gathered}
$$

В случае бесконечномерного пространства $H^{\mathrm{cl}}$ характеристический функционал есть интеграл по бесконечномерному фазовому пространству. Как это принято в статистической механике, в этой ситуации производится конечномерная аппроксимация интеграла, при которой в суммах $\sum_{a} Y_{a}^{\mathrm{cl}}(0) u_{a} \equiv Y^{\mathrm{cl}}(0) u$ оставляют конечное число переменных интегрирования, а затем осуществляется предельный переход к бесконечному множеству переменных $Y_{a}^{\mathrm{cl}}(0)$. В данном случае эта предельная процедура тривиальна, и ее результат суть формулы (35) или (36). Формулы (36) однозначно определяют векторный гауссовский случайный процесс $Y^{\mathrm{cl}}(t)$ над $H^{\mathrm{cl}}$ с характеристическим функционалом

$$
\left\langle\exp \left(i \sum_{j=1}^{n} Y^{\mathrm{cl}}\left(t_{j}\right) u_{j}\right)\right\rangle^{\mathrm{cl}}=\exp \left(-\sum_{j, k=1}^{n} \frac{\left(u_{j} \mid W^{\mathrm{cl}}\left(t_{j}-t_{k}\right) u_{k}\right)^{\mathrm{cl}}}{2}\right),
$$

где $\left\{t_{j}\right\}$ и $\left\{u_{j}\right\}$ - произвольные конечные наборы из $\mathbb{R}$ и из $\chi$.

Формулы (35) показывают, что $Y^{\mathrm{cl}}(t)$, как векторный гауссовский случайный процесс над $H^{\mathrm{cl}}$, действительно может быть отождествлен с $Y^{\mathrm{cl}} U(t)$ в соответствии с предписанием (27).

Открытая система должна входить в полную систему. Имея это в виду, динамическим переменным $X(t) l$ открытой системы сопоставим переменные $\widehat{X}(t) l$ из алгебры полной системы, полагая

$$
\widehat{X}(t) l=Y^{\mathrm{cl}} x(t) l
$$

или в сглаженной форме

$$
\widehat{X}[\Phi]=Y^{\mathrm{cl}} x[\Phi] .
$$

Убедимся в совпадении корреляционных функций исходных и новых величин, используя (28) (при $u=x[\Phi], \nu=x[\Psi])$ и (14):

$$
\langle\widehat{X}[\Phi] \cdot \widehat{X}[\Psi]\rangle^{\mathrm{cl}}=\beta^{-1}(x[\Phi] \mid x[\Phi])^{\mathrm{cl}}=\langle X[\Phi] \cdot X[\Psi]\rangle^{\mathrm{cl}} .
$$

Учтем, что рассматриваемые случайные величины гауссовы с нулевым средним. Это позволяет отождествить $\widehat{X}[\Phi]$ с $X[\Phi]$, а $\widehat{X}(t)$ с $X(t)$ и не делать различия между 
отождествленными величинами. Тем самым переменные открытой системы становятся частью алгебры полной системы, и исходный процесс есть результат сужения состояния полной системы на алгебру открытой системы.

Докажем теперь формулу (3) с помощью (39) (где $\left.Y^{\mathrm{cl}}=Y^{\mathrm{cl}}(0)\right)$ и $(29)($ при $t=0)$ :

$$
\begin{aligned}
\left\{\widehat{X}(s) l_{1}, \widehat{X}(t) l_{2}\right\} & =-\left(D \chi(s) l_{1} \mid \chi(t) l_{2}\right)^{\mathrm{cl}}=-\left.\left(\partial_{\tau} U(\tau) \chi(s) l_{1} \mid \chi(t) l_{2}\right)^{\mathrm{cl}}\right|_{\tau=0}= \\
& =-\left.\partial_{\tau}\left(\chi(s+\tau) l_{1} \mid \chi(t) l_{2}\right)^{\mathrm{cl}}\right|_{\tau=0}=-\left.\beta \cdot \partial_{\tau}\left\langle X(s+\tau) l_{1} \cdot X(t) l_{2}\right\rangle^{\mathrm{cl}}\right|_{\tau=0} .
\end{aligned}
$$

Это означает справедливость формулы (3).

2.3. Квантование полной системы. Сначала выполним квантование полной системы. В согласии с принципом соответствия вместо классических случайных величин $Y^{\mathrm{cl}}(t) u$ (из п. 2.2) мы вводим теперь квантовые случайные величины $Y^{\mathrm{qu}}(t) u$, $u \in \chi$. В силу линейности системы зависимость от времени, как и в классическом случае, определяется соотношением типа $(27): Y^{\mathrm{qu}}(t)=Y^{\mathrm{qu}}(0) U(t)$, или

$$
Y^{\mathrm{qu}}(t)=Y^{\mathrm{qu}} U(t)
$$

Скобке Пуассона (29) соответствуют канонические коммутационные соотношения $(\mathrm{KKC})$ :

$$
\left[Y^{\mathrm{qu}}(s) u, Y^{\mathrm{qu}}(t) \nu\right]=-i \hbar(u \mid \mathcal{D}(s-t) \nu)^{\mathrm{cl}}, \quad u, \nu \in \chi,
$$

где под знаком коммутатора фигурируют эрмитовы операторы в комплексном гильбертовом пространстве $\mathcal{H}^{\text {qu }}$ представления КKC.

В квантовой теории поля операторы рождения и уничтожения определяются как отрицательно- и положительно-частотные части полевых операторов. В данном случае это $\widetilde{Y}^{(-)}(\lambda)=\widetilde{Y}^{\text {qu }}(\lambda) \cdot \theta(-\lambda)$ и $\widetilde{Y}^{(+)}(\lambda)=\widetilde{Y}^{\text {qu }}(\lambda) \cdot \theta(\lambda)$, где $\theta(\lambda)-$ функция Хевисайда (см. пояснение к формуле (6)). Отсюда находим двухточечную функцию вакуумного состояния квантовой полной системы (при $T=0)$ :

$$
\left\langle Y^{\mathrm{qu}}(s) u \cdot Y^{\mathrm{qu}}(t) \nu\right\rangle_{0}^{\mathrm{qu}}=\left[Y^{(+)}(s) u, Y^{(-)}(t) \nu\right] \equiv\left(u \mid W_{0}^{\mathrm{qu}}(s-t) \nu\right)^{\mathrm{cl}},
$$

где

$$
\widetilde{W}_{0}^{\mathrm{qu}}(\lambda):=-i \hbar \theta(\lambda) \widetilde{\mathcal{D}}(\lambda)=(\beta \hbar \lambda)_{+} \widetilde{W}^{\mathrm{cl}}(\lambda) .
$$

Аналогично температурная двухточечная функция гиббсовского состояния квантовой полной системы

$$
\left\langle Y^{\mathrm{qu}}(s) u \cdot Y^{\mathrm{qu}}(t) \nu\right\rangle^{\mathrm{qu}} \equiv\left(u \mid W^{\mathrm{qu}}(s-t) \nu\right)^{\mathrm{cl}}
$$

восстанавливается по перестановочной функции по формуле ${ }^{5)}$

$$
\widetilde{W}^{\mathrm{qu}}(\lambda)=-\frac{i \hbar}{2}\left(\operatorname{cth} \frac{\beta \hbar \lambda}{2}+1\right) \widetilde{\mathcal{D}}(\lambda),
$$

или

$$
\widetilde{W}^{\mathrm{qu}}(\lambda)=\frac{\beta \hbar \lambda}{2}\left(\operatorname{cth} \frac{\beta \hbar \lambda}{2}+1\right) \widetilde{W}^{\mathrm{cl}}(\lambda) .
$$

5) По поводу вывода формулы (46) см., например, [1], приложение 2. Отправной точкой служит правило фон Неймана для квантового среднего $\langle A\rangle^{\mathrm{qu}}:=Q^{-1} \cdot \operatorname{tr}\left(A e^{-\beta H}\right)$, где $Q:=\operatorname{tr} e^{-\beta H}$, динамической переменной $A$ конечномерной канонической системы в гиббсовском состоянии. 
Из (45) вытекает

$$
\left\langle Y^{\mathrm{qu}}(s) u \cdot Y^{\mathrm{qu}}(t) \nu\right\rangle^{\mathrm{qu}}=\mathrm{B}\left(i \beta \hbar \partial_{s}\right)\left\langle Y^{\mathrm{cl}}(s) u \cdot Y^{\mathrm{cl}}(t) \nu\right\rangle^{\mathrm{cl}} .
$$

В частности, используя (31), (41), получаем

$$
\left\langle Y^{\mathrm{qu}} u \cdot Y^{\mathrm{qu}} \nu\right\rangle^{\mathrm{qu}}=\left.\mathrm{B}\left(i \beta \hbar \partial_{\tau}\right)\left\langle Y^{\mathrm{cl}} U(\tau) u \cdot Y^{\mathrm{cl}} \nu\right\rangle^{\mathrm{cl}}\right|_{\tau=0}
$$

Известно (см. [17], гл. XII, §12), что в случае квадратичного гамильтониана без линейного члена для гиббсовского состояния имеет место формула

$$
\left\langle e^{i \xi}\right\rangle^{\mathrm{qu}}=e^{-\left\langle\xi^{2}\right\rangle^{\mathrm{qu}} / 2}
$$

где $\xi$ - произвольная линейная комбинация динамических переменных (в данном случае $\left.Y_{a}^{\mathrm{qu}}(0)\right)$. Отсюда следует, что гиббсовское состояние с температурой $T$ полной системы, как и вакуумное состояние, является гауссовским с характеристическим функционалом

$$
\left\langle\exp \left(i \sum_{j=1}^{n} Y^{\mathrm{qu}}\left(t_{j}\right) u_{j}\right)\right\rangle^{\mathrm{qu}}=\exp \left(-\sum_{j, k=1}^{n} \frac{\left(u_{j} \mid W^{\mathrm{qu}}\left(t_{j}-t_{k}\right) u_{k}\right)^{\mathrm{cl}}}{2}\right),
$$

где $\left\{t_{j}\right\},\left\{u_{j}\right\}$ - произвольные конечные наборы из $\mathbb{R}$ и из $\chi$. (Гауссовские состояния алгебры KKC называют также квазисвободными [18]-[20].)

2.4. Квантование открытой системы. Остается произвести сужение квантового состояния на подалгебру, порожденную переменными $X(t) l$. Согласно формуле (41) $Y^{\mathrm{qu}}(0) u=Y^{\mathrm{qu}} u$. В частности, вектору $u=x[\Phi] \in \chi$ при квантовании сопоставляется оператор $Y^{\mathrm{qu}} u$. Соответственно, вектору $\int x(t) l \cdot \varphi(t) d t \in \chi$ сопоставляется оператор $Y^{\text {qu }} \int x(t) l \cdot \varphi(t) d t-$ квантовый аналог (классической) случайной величины

$$
Y^{\mathrm{cl}} \int x(t) l \cdot \varphi(t) d t \equiv \int X(t) l \cdot \varphi(t) d t .
$$

Таким образом, квантование открытой системы сводится к замене классической величины $X(t) l \equiv Y^{\mathrm{cl}} x(t) l$ на квантовую

$$
X(t) l=Y^{\mathrm{qu}} x(t) l .
$$

Здесь $Y^{\text {qu }}$ берется из квантовой реализации полной системы (п. 2.3), а выражение $x(t) l$, сглаженное с основной функцией по $t$, является вектором из пространства $H^{\mathrm{cl}}$ классического процесса.

Очевидно, квантовое состояние - это также гауссовское состояние с нулевым средним. Двухточечная функция квантового векторного процесса $X(t)$ находится из соотношений (51), (48):

$$
\begin{aligned}
\left\langle X(s) l_{1} \cdot x(t) l_{2}\right\rangle^{\mathrm{qu}} & =\left\langle Y^{\mathrm{qu}} x(s) l_{1} \cdot Y^{\mathrm{qu}} x(t) l_{2}\right\rangle^{\mathrm{qu}}= \\
& =\left.\mathrm{B}\left(i \beta \hbar \partial_{\tau}\right)\left\langle Y^{\mathrm{cl}} U(\tau) x(s) l_{1} \cdot Y^{\mathrm{cl}} x(t) l_{2}\right\rangle^{\mathrm{cl}}\right|_{\tau=0}= \\
& =\mathrm{B}\left(i \beta \hbar \partial_{s}\right)\left\langle X(s) l_{1} \cdot X(t) l_{2}\right\rangle^{\mathrm{cl}},
\end{aligned}
$$

что совпадает с формулой (7). Это завершает процедуру квантования открытой системы в соответствии с концепцией гамильтонова моделирования и вывод формулы (7). Формула (6) получается из (7) в пределе $T \rightarrow 0$. 
ЗАМЕчАНИЕ 1. Выше на исходный процесс налагалось условие (21), исключавшее "нулевые моды". Теперь, полагая, что $T>0$, мы распространим результаты на процессы, для которых условие (21) не выполнено, т. е. мы предполагаем, что линеал (т.е. линейное подпространство)

$$
H_{\mathrm{st}}:=\left\{u \in H^{\mathrm{cl}}: U(\tau) u=u \quad \text { при всех } \quad \tau \in \mathbb{R}\right\} \equiv D^{-1}\{0\}
$$

отличен от $\{0\}$. С этой целью мы аппроксимируем исходный процесс $X(t)$ процессом $X^{(\gamma)}(t)$ над $L$, в котором условие $(21)$ выполнено. Обозначим через $\mathbf{P}_{\mathrm{st}}$ и $\mathbf{P}_{\mathrm{st}}^{\perp}$ ортогональные проекторы в $H^{\mathrm{cl}}$ на замкнутый линеал $H_{\mathrm{st}}$ и на ортогональное дополнение $H_{\mathrm{st}}^{\perp}$. В соответствии с формулой (14) двухточечная функция исходного процесса над $L$ разлагается в сумму

$$
\left\langle X(s) l_{1} \cdot X(t) l_{2}\right\rangle^{\mathrm{cl}}=\beta^{-1}\left(x(s) l_{1} \mid \mathbf{P}_{\mathrm{st}} x(t) l_{2}\right)^{\mathrm{cl}}+\beta^{-1}\left(x(s) l_{1} \mid \mathbf{P}_{\mathrm{st}}^{\perp} x(t) l_{2}\right)^{\mathrm{cl}},
$$

где первое слагаемое не зависит от $s-t$, так что его преобразование Фурье (от переменной $s-t$ к $\lambda$ ) пропорционально $\delta(\lambda)$. При $\gamma>0$ определим двухточечную функцию векторного гауссовского случайного процесса $X^{(\gamma)}(t)$ над $L$ формулой

$$
\left\langle X^{(\gamma)}(s) l_{1} \cdot X^{(\gamma)}(t) l_{2}\right\rangle^{\mathrm{cl}}=\beta^{-1}\left(x(s) l_{1} \mid \mathbf{P}_{\mathrm{st}} x(t) l_{2}\right)^{\mathrm{cl}} e^{-\gamma|s-t|}+\beta^{-1}\left(x(s) l_{1} \mid \mathbf{P}_{\mathrm{st}}^{\perp} x(t) l_{2}\right)^{\mathrm{cl}} .
$$

При $\gamma>0$ условие (21) выполнено, так как вклады, пропорциональные $\delta(\lambda)$, отсутствуют. Теперь в основных формулах п. 1.3 можно подставить $X^{(\gamma)}(t)$ вместо $X(t)$. Переходя в этих формулах к пределу $\gamma \rightarrow 0$, мы переносим процедуру квантования (из п. 1.3) на общий случай.

Отметим также, что для кососимметрического оператора $D$ вместо формулы (23) теперь мы имеем разложение

$$
D=0 \oplus J \Omega
$$

в соответствии с представлением $H^{\mathrm{cl}}=H_{\mathrm{st}} \oplus H_{\mathrm{st}}^{\perp}$. По-прежнему $J^{*}=-J, J^{2}=-1$, $\Omega J=J \Omega, \Omega>0$. Попутно заметим, что квантовую двухточечную функцию (7) можно записать с помощью операторов $\mathbf{Q}$ и $\hbar D / 2$ в $H^{\mathrm{cl}}$ :

$$
\left\langle X(s) l_{1} \cdot X(t) l_{2}\right\rangle^{\mathrm{qu}}=\beta^{-1}\left(x(s) l_{1} \mid \mathbf{Q} x(t) l_{2}\right)^{\mathrm{cl}}-i\left(x(s) l_{1} \mid \frac{\hbar D}{2} x(t) l_{2}\right)^{\mathrm{cl}}
$$

где $\mathbf{Q} \geqslant 1$ - положительный оператор в $H^{\mathrm{cl}}$, коммутирующий с представлением $U(\tau)$ и с областью определения, как у $D$ :

$$
\mathbf{Q}=1 \oplus \frac{\beta \hbar D}{2} \operatorname{ctg} \frac{\beta \hbar D}{2}=1 \oplus \frac{\beta \hbar \Omega}{2} \operatorname{cth} \frac{\beta \hbar \Omega}{2} .
$$

При условии $H_{\mathrm{st}} \neq\{0\}$ мы по-прежнему можем называть состояние открытой системы, описываемой векторным случайным процессом $X(t)$, гиббсовским (хотя и в расширительном значении).

Как следует из формул (53), (55), при квантовании с температурой $T>0$ "нулевые моды" остаются классическими в том смысле, что "стационарная компонента" $X_{\mathrm{st}}(t) l:=Y^{\mathrm{cl} / \mathrm{qu}} \mathbf{P}_{\mathrm{st}} x(t) l$ процесса $X(t) l$ удовлетворяет соотношениям

$$
\left\langle X_{\mathrm{st}}(s) l_{1} \cdot X(t) l_{2}\right\rangle^{\mathrm{qu}}=\left\langle X_{\mathrm{st}}(s) l_{1} \cdot X(t) l_{2}\right\rangle^{\mathrm{cl}}, \quad\left\{X_{\mathrm{st}}(s) l_{1}, X(t) l_{2}\right\}=0 .
$$


Физическим примером гауссовского процесса с "нулевой модой" служит модель нерелятивистского бесконечного свободного бозе-газа при нулевом химическом потенциале. Если формулу (4.9) из работы [21] проинтегрировать по $\rho_{0}$ с подходящей функцией (как в (5.3) для $T=0$ ), то получится гауссовское состояние системы ККС, в котором "нулевая мода" создает дополнительный вклад в плотность числа частиц. Это явление носит название конденсации Бозе-Эйнштейна ([16], §62).

ЗАмЕчАниЕ 2. В рассматриваемом классе стационарных гауссовских (обобщенных) случайных процессов линейные (в частности, стохастические) дифференциальные уравнения с постоянными коэффициентами, верные в классической области, справедливы и в квантовой области. Очевидно, что такие уравнения устанавливают связи между корреляционными функциями входящих в уравнение различных случайных процессов. Например, процессы осцилляторного броуновского движения по Эйнштейну и по Ланжевену удовлетворяют соответственно уравнениям

$$
\begin{aligned}
m \gamma \partial_{t} q(t)+m \omega_{0}^{2} q(t) & =\sqrt{2 m \gamma} \eta(t), \\
m \partial_{t}^{2} q(t)+m \gamma \partial_{t} q(t)+m \omega_{0}^{2} q(t) & =\sqrt{2 m \gamma} \eta(t),
\end{aligned}
$$

где $q(t)$ - координата частицы с массой $m, m \gamma$ - коэффициент вязкого трения, $\sqrt{2 m \gamma} \eta(t)$ - флуктуирующая сила, $\eta(t)$ - белый шум с интенсивностью $\beta^{-1}=k T$, являющийся гауссовским обобщенным случайным процессом с нулевым средним и с двухточечной функцией

$$
\langle\eta(s) \cdot \eta(t)\rangle^{\mathrm{cl}}=\beta^{-1} \delta(s-t) .
$$

В квантовой области имеют место те же уравнения, где $\eta(t)$ - квантовый белый шум,

$$
\begin{gathered}
{[\eta(s), \eta(t)]=i \hbar \delta^{\prime}(s-t),} \\
\langle\eta(s) \cdot \eta(t)\rangle^{\mathrm{qu}}=\int \mathrm{B}(\beta \hbar \lambda) e^{i \lambda(s-t)} \frac{d \lambda}{2 \pi} .
\end{gathered}
$$

Гамильтоново моделирование в конструктивном методе имеется для уравнения (59) (см., например, [6]) и отсутствует для уравнения (58). Изложенный выше метод гамильтонова моделирования и, как следствие, квантования применим к обоим уравнениям (58), (59).

\section{3. КВАНТОВАНИЕ ЛИНЕЙНЫХ СИСТЕМ С ИНФРАКРАСНЫМИ ОСОБЕННОСТЯМИ ДВУХТОЧЕЧНЫХ ФУНКЦИЙ}

3.1. Стационарное виртуальное состояние открытой системы. В этом разделе мы применим метод гауссовских потоков для гамильтонова моделирования открытых систем, взаимодействующих с термостатом, но не находящихся в термодинамическом равновесии. Примером такой системы является свободная броуновская частица, у которой стационарное состояние отсутствует вследствие симметрии законов движения относительно пространственных трансляций.

Удобным средством, не нарушающим симметрий, присущих таким системам, является понятие стационарного гауссовского виртуального состояния *-алгебры системы. Оно отличается от физического состояния тем, что условие положительности $\left\langle A^{2}\right\rangle^{\mathrm{cl}} \geqslant 0$ на вещественных элементах алгебры системы, вообще говоря, не 
выполняется. Ясно, что виртуальное состояние не имеет самостоятельного значения и лишь служит средством для построения физических состояний системы ${ }^{6)}$. Для описания виртуального процесса $X(t)$, соответствующего виртуальному состоянию, используется формализм пространств с индефинитной метрикой. Существенной чертой формализма, отличающей его от стандартных трактовок, является явная инвариантность относительно временных трансляций и, возможно, относительно обращения времени (например, в модели свободного броуновского движения). Это обстоятельство существенно для цели квантования.

При определенных условиях виртуальное состояние позволяет построить стационарное обобщенное состояние (с бесконечной нормой) вырожденного гауссовского типа - в смысле предела ненормированных гауссовских положительных функционалов. (Простейшим примером обобщенного состояния служит интеграл от функций на вещественной прямой $\mathbb{R}$ по мере Лебега; роль алгебры системы играет некоторая алгебра функций на $\mathbb{R}$.)

С помощью конструкции типа процедуры Гельфанда-Наймарка-Сигала (ГНС) [22] из стационарного обобщенного состояния можно "приготовить" бесконечный класс нестационарных (не обязательно гауссовских) состояний одной и той же физической системы при одних и тех же внешних условиях. Стационарное обобщенное состояние оказывается эффективным способом организации нестационарных состояний системы. (В контексте квантовой теории поля "при нулевой температуре" перечисленные этапы подробно описаны в [23], гл. 11.)

ЗАмЕчАниЕ 3. Сравним для примера излагаемый подход с традиционной картиной классического свободного броуновского движения (по Эйнштейну), которая представляет не связанные друг с другом случайные процессы $q(t), t \geqslant t_{0}$, отличающиеся начальным значением времени $t_{0}$ и начальным условием. Теперь же мы имеем дело с множеством физических состояний единой алгебры динамических переменных $q(t)$ при всех $t \in \mathbb{R}$, удовлетворяющих уравнению (58) при $\omega_{0}=0$ и первому из уравнений (61). Особый интерес представляют состояния, "приготовленные" на отрезке времени $\left[s_{0}, t_{0}\right]$ из стационарного обобщенного состояния $s^{\mathrm{cl}}$. Они имеют вид $s_{B}^{\mathrm{cl}}(A)=s^{\mathrm{cl}}\left(B^{*} A B\right)$, где $B$ - элемент подалгебры, порожденной переменными $q(t)$ при $t \in\left[s_{0}, t_{0}\right]$, нормированный условием $s^{\mathrm{cl}}\left(B^{*} B\right)=1$. (Специфика этого "приготовления" состояния состоит в том, что оно формирует не только будущее, но и прошлое. Это следствие того, что в теории "приготовлению" состояния предшествует этап конструкции стационарного состояния $s^{\mathrm{cl}}$.) Условие (60), справедливое для виртуального процесса, выполняется для физического процесса лишь при $s, t$, не принадлежащих отрезку $\left[s_{0}, t_{0}\right]$ времени “приготовления" состояния из $s^{\mathrm{cl}}$. Соответственно, условие марковости для $q(t)$ (в классическом режиме) выполняется при $t \geqslant t_{0}$ (в зависимости от состояния), а при $t \leqslant s_{0}$ выполняется условие марковости в обращенном времени. В результате мы получаем обратимое во времени гамильтоново описание свободного броуновского движения.

3.2. Исходные положения и некоторые результаты. Как и в разделе 2, ставится задача построения классической полной системы для данной открытой системы и последующего квантования ее. Исходные положения частично совпадают с изложенными в разделе 2. Вместо векторного стационарного гауссовского

6) Тем не менее, как показано в [14], асимптотика при $t \rightarrow \infty$ виртуальной двухточечной функции свободного броуновского движения входит в асимптотическую формулу для корреляций в физических состояниях как классического, так и квантового температурного представления. 
процесса над $L$ теперь для описания эволюции классической открытой системы во взаимодействии с термостатом используется векторный стационарный гауссовский виртуальный процесс $X(t)$ над вещественным векторным пространством $L$, определяемый следующим образом.

Имеется вещественное гильбертово пространство $H^{\mathrm{cl}}$ со скалярным произведением $(u, \nu)$ векторов $u, \nu$. Пусть $Q$ - гауссовская векторная случайная величина над $H^{\mathrm{cl}}$, которая сопоставляет линейным образом векторам $u \in H^{\mathrm{cl}}$ гауссовские случайные величины $Q u$ с нулевым средним и с ковариацией $\operatorname{cov}(Q u, Q \nu)=\beta^{-1}(u, \nu)$, где $\beta=1 / k T, u, \nu \in H^{\mathrm{cl}}$. Кроме того, в $H^{\mathrm{cl}}$ определен линейный самосопряженный ограниченный оператор $\theta$ с ограниченным обратным, который задает в $H^{\text {cl }}$ знаконеопределенную симметрическую билинейную форму $(u \mid \nu)^{\mathrm{cl}}:=(u, \theta \nu)$. Функционал $e^{-(u \mid u)^{\mathrm{cl}} / 2 \beta}$ от $u \in H^{\mathrm{cl}}$ интерпретируется как виртуалъное среднее от величины $e^{i Q u}$ и обозначается как

$$
\left\langle e^{i Q u}\right\rangle^{\mathrm{cl}}:=e^{-(u \mid u)^{\mathrm{cl}} / 2 \beta}
$$

Аналогично определяются виртуалъные средние произведений $Q u_{1}, \ldots, Q u_{n}$ :

$$
\left\langle Q u_{1} \ldots Q u_{n}\right\rangle^{\mathrm{cl}}:=\left.(-i)^{n} \partial_{\alpha_{1}} \ldots \partial_{\alpha_{n}}\left\langle e^{i \alpha_{1} Q u_{1}+\cdots+i \alpha_{n} Q u_{n}}\right\rangle^{\mathrm{cl}}\right|_{\alpha_{1}=\cdots=\alpha_{n}=0} .
$$

Случайные величины с таким образом определенными виртуальными средними называются гауссовскими виртуальными величинами.

Векторным гауссовским (обобщенным) виртуальным процессом $X(t)$ над $L$ с нулевым средним называется пара билинейных отображений, сопоставляющих элементам $(\varphi, l) \in S_{\mathrm{r}}(\mathbb{R}) \times L$ вектор $u=\int x(t) l \cdot \varphi(t) d t \in H^{\mathrm{cl}}$, непрерывно зависящий от $\varphi \in S_{\mathrm{r}}(\mathbb{R})$, и гауссовскую виртуальную величину $Q u$, которую мы обозначаем посредством $\int X(t) l \varphi(t) d t$.

Пусть

$$
\xi=\sum_{j} \int X(t) l_{j} \varphi_{j}(t) d t
$$

- произвольная конечная сумма. Комплексные (конечные) линейные комбинации $A=\sum_{\xi} c_{\xi} e^{i \xi}$ величин $e^{i \xi}$ образуют *-алгебру открытой системы. Buртуалъным состоянием этой $*$-алгебры называется линейный функционал

$$
A \mapsto\langle A\rangle^{\mathrm{cl}}=\sum_{\xi} c_{\xi}\left\langle e^{i \xi}\right\rangle^{\mathrm{cl}} .
$$

В результате вся информация о стационарном гауссовском виртуальном состоянии с нулевым средним *-алгебры открытой системы содержится в классических одноточечной и двухточечной функциях вида

$$
\begin{aligned}
\langle X(t) l\rangle^{\mathrm{cl}} & =0, \quad l \in L, \\
\left\langle X(s) l_{1} \cdot X(t) l_{2}\right\rangle^{\mathrm{cl}} & =\beta^{-1} C\left(s-t ; l_{1}, l_{2}\right), \quad l_{1}, l_{2} \in L,
\end{aligned}
$$

где $C\left(s-t ; l_{1}, l_{2}\right)$ - вещественная билинейная форма от векторов $l_{1}, l_{2} \in L$, инвариантная при перестановке $\left(s, l_{1}\right) \leftrightarrow\left(t, l_{2}\right)$, зависящая от $s-t$ как обобщенная функция умеренного роста. 
Для гамильтонова моделирования полной системы и последующего квантования ее при конечной температуре ${ }^{7)}$ мы налагаем на двухточечную функцию дополнительные ограничения. Они формулируются в виде условий 1-4 и обсуждаются в п. 3.4-3.6. Как и в разделе 2, результатом гамильтонова моделирования оказывается возможность построения полной системы с эволюцией типа (31) для векторной динамической переменной $Y(t)$. Для скобок Пуассона и коммутаторов принимаются прежние выражения (3), (4). Что касается формулы (7), то она задает двухточечную функцию теперь уже квантового виртуального состояния открытой системы при конечной температуре.

Для перехода к физическим состояниям системы при фиксированной температуре достаточно построить физическое температурное представление *-алгебры полной системы. Тогда физические состояния получаются как состояния, ассоциированные с этим представлением. Вначале мы строим физическое представление для калибровочно-инвариантных величин. Из условий 1-4 вытекает существование переменной $z_{\xi}$, которая сохраняется во времени и называется зарядом. Она является линейным функционалом от переменной $\xi(62)$ и принимает значения в вещественном конечномерном пространстве $\mathcal{Z}(\operatorname{dim} \mathcal{Z}=n \geqslant 1)$, а именно

$$
z_{\xi}:=\sum_{j} \tilde{\varphi}_{j}(0) \cdot \zeta l_{j},
$$

где $\zeta: L \rightarrow \mathcal{Z}$ есть линейное отображение $L$ на $\mathcal{Z}$ (см. следствие 4 в п. 3.6). Линейное пространство $G$, сопряженное к $\mathcal{Z}$, назовем группой калибровочных преобразований $\xi \mapsto \xi+g \cdot z_{\xi}$

Элемент *-алгебры вида $\sum_{\xi} c_{\xi} e^{i \xi}$ является калибровочно-инвариантным, если в сумме присутствуют лишь слагаемые с $z_{\xi}=0$. На таких $\xi$ выполнено условие положительности в виде $\left\langle\xi^{2}\right\rangle^{\mathrm{cl}} \geqslant 0$. Поэтому к подалгебре калибровочно-инвариантных величин можно применить результаты раздела 2 и получить классическое и, соответственно, квантовое гиббсовское состояние $s_{0}^{\sharp}$ подсистемы с характеристическим функционалом

$$
s_{0}^{\sharp}\left(e^{i \xi}\right)=e^{-\left\langle\xi^{2}\right\rangle^{\sharp} / 2} \quad \text { при } \quad z_{\xi}=0,
$$

где индекс $\sharp$ означает “cl” или “qu”, а квантовая двухточечная функция по-прежнему определена, как в (7). Процедура ГНС позволяет построить представление $\pi_{0}^{\sharp} *$-алгебры, порожденной калибровочно-инвариантными элементами $e^{i \xi}$, в некотором гильбертовом пространстве $\mathcal{H}_{0}^{\sharp}$ с циклическим вектором $\mid$ GIBBS $\rangle^{\sharp}$.

Для построения температурного представления $\pi^{\sharp}$ воспользуемся понятием индуцированного представления группы по представлению ее подгруппы ([25], гл. 16, § 1). Здесь ссылка на группу уместна, так как *-алгебра открытой системы порождается унитарными элементами $e^{i(\xi+a)}$, а элементы $\xi \oplus a$, где $a \in \mathbb{R}$, образуют группу G. (В классическом случае $\mathrm{G}$ - абелева группа по сложению, а в квантовом случае $\mathrm{G}$ - группа Гейзенберга-Вейля.) Калибровочно-инвариантные элементы образуют подгруппу $\mathrm{G}_{0}$, причем $\mathrm{G} / \mathrm{G}_{0} \approx \mathcal{Z}$. Поскольку известно унитарное представление подгруппы $\mathrm{G}_{0}$ как сужение $\pi_{0}^{\sharp}$ на элементы $e^{i(\xi+a)}$, то мы можем получить

\footnotetext{
7) Вакуумные состояния алгебры квантовой полной системы, соответствующие нулевой температуре, мы здесь не рассматриваем (по этому поводу см. [24]).
}

6 Теоретическая и математическая физика, т. 173, № 3, 2012 г. 
индуцированное представление группы G. В результате получаем формулу для характеристического функционала стационарного обобщенного состояния

$$
s^{\sharp}\left(e^{i \xi}\right)=(2 \pi)^{n} \delta\left(z_{\xi}\right) e^{-\left\langle\xi^{2}\right\rangle^{\sharp} / 2}
$$

и температурное представление $\pi^{\sharp}$ *-алгебры открытой системы.

Следствие 6 (см. ниже п. 3.6) и отчасти формулы (64), (65) показывают, что в нашем случае индефинитность скалярного произведения $(u \mid \nu)^{\mathrm{cl}}$ обусловлена инфракрасными особенностями двухточечной функции. Конечномерность ранга индефинитности $n$ означает, что отклонения от теплового равновесия физических состояний, ассоциированных с представлением $\pi^{\sharp}$, носят квазилокальный характер, и для полной системы сохраняет смысл понятие температуры. Ниже мы обсудим это и другие следствия, которые вытекают из условий 1-4 (см. п. 3.4-3.6). Температурное представление *-алгебры полной системы получено в п. 3.10, а его свойства разбираются в п. 3.11. Там (в отличие от настоящего пункта) рассмотрение ведется в основном в терминах *-алгебры и динамических переменных полной системы. Реализация переменных открытой системы операторами температурного представления полной системы дается формулой (132).

В качестве эталонных моделей скалярных стационарных гауссовских виртуальных процессов, попадающих в рассматриваемый класс процессов (при $L=\mathbb{R}$ ), следует выделить семейство фракталъных (или дробных) броуновских движений $q(t)$. Такой виртуальный процесс характеризуется следующими одноточечной и двухточечной функциями:

$$
\langle q(t)\rangle^{\mathrm{cl}}=0, \quad\langle q(s) \cdot q(t)\rangle^{\mathrm{cl}}=-\frac{d(\alpha)}{\beta}|s-t|^{2 \alpha} .
$$

Здесь $\alpha$ - параметр Херста $(0<\alpha<1), d(\alpha)$ - размерный множитель. Значение $\alpha=1 / 2$ соответствует обычному броуновскому движению с константой диффузии $\mathbf{D}=d(1 / 2) / \beta=1 / m \gamma \beta$.

3.3. Свойства виртуальной двухточечной функции. Фундаментальным отличием двухточечной функции виртуального процесса от обычной является то, что она не удовлетворяет условию положительности, а именно: "виртуальное среднее"

$$
\left\langle\left(\sum_{j=1}^{N} \int X(t) l_{j} \cdot \varphi_{j}(t) d t\right)^{2}\right\rangle^{\mathrm{cl}}
$$

принимает все вещественные значения при $N=1,2, \ldots, \varphi_{j} \in S_{\mathrm{r}}(\mathbb{R}), l_{j} \in L, j=$ $1, \ldots, N$ (ср. с соотношением (12) из раздела 2). Использование здесь и далее сглаженных величин $\int X(t) l \cdot \varphi(t) d t$ позволяет включить в рассмотрение обобщенные динамические переменные (в смысле теории обобщенных функций). Для такой сглаженной величины мы используем обозначение $X[\varphi \otimes l]$.

ЗАмЕчАниЕ 4. Существуют различные способы построения двухточечной функции "виртуального процесса". Так, формула (3) позволяет подобрать виртуальную 
двухточечной функцию, если известны скобки Пуассона для $X(t) l$ (при этом следует убедиться в выполнении дополнительных условий 1-4 из п. 3.4-3.6.) Из линейных стохастических дифференциальных уравнений с постоянными коэффициентами также можно находить двухточечную функцию. Например, виртуальная двухточечная функция свободного броуновского движения находится из уравнений (58) или (59) (при $\left.\omega_{0}=0\right)$ и первого из уравнений (61) (см. [14], раздел 2).

Преобразование Фурье $\widetilde{C}\left(\lambda ; l_{1}, l_{2}\right):=F_{t \rightarrow \lambda}\left[C\left(t ; l_{1}, l_{2}\right)\right]$ (от переменной $t$ к переменной $\lambda)$ для $C\left(t ; l_{1}, l_{2}\right)$ обладает следующими свойствами:

$$
\widetilde{C}\left(-\lambda ; l_{1}, l_{2}\right)=\overline{\widetilde{C}}\left(\lambda ; l_{1}, l_{2}\right)=\widetilde{C}\left(\lambda ; l_{2}, l_{1}\right) .
$$

Суммы векторов вида $\varphi \otimes l$, где $\varphi \in S_{\mathrm{r}}(\mathbb{R}), l \in L$, образуют тензорное произведение $\mathbf{S}:=S_{\mathrm{r}}(\mathbb{R}) \otimes L$. По линейности определены величины $X[\Phi]$ при любых $\Phi:=\sum_{j} \varphi_{j} \otimes l_{j}$ из $\mathbf{S}$. В новых обозначениях двухточечная функция (63), сглаженная с $\varphi(s) \cdot \psi(t)$ по $s, t$, записывается как $\left\langle X\left[\varphi \otimes l_{1}\right] \cdot X\left[\psi \otimes l_{2}\right]\right\rangle^{\text {cl }}$. По билинейности на $\mathbf{S}$ определена непрерывная билинейная симметрическая форма

$$
\beta \cdot\langle X[\Phi] \cdot X[\Psi]\rangle^{\mathrm{cl}}, \quad \Phi, \Psi \in \mathbf{S} .
$$

Если $\Phi=\sum_{j} \varphi_{j} \otimes l_{j}, \Psi:=\sum_{k} \psi_{k} \otimes l_{k}^{\prime}$, то

$$
\beta \cdot\langle X[\Phi] \cdot X[\Psi]\rangle^{\mathrm{cl}}=\int \widetilde{C}(\lambda \mid \Phi, \Psi) \frac{d \lambda}{2 \pi},
$$

где

$$
\widetilde{C}(\lambda \mid \Phi, \Psi):=\sum_{j, k} \widetilde{C}\left(\lambda ; l_{j}, l_{k}^{\prime}\right) \overline{\tilde{\varphi}}_{j}(\lambda) \tilde{\psi}_{k}(\lambda) .
$$

Изотропный линеал (т. е. изотропное линейное подпространство) $\mathbf{S}^{\perp}$ в $\mathbf{S}$ определяется посредством

$$
\mathbf{S}^{\perp}:=\left\{\Phi \in \mathbf{S}: \beta \cdot\langle X[\Psi] \cdot X[\Phi]\rangle^{\mathrm{cl}}=0 \text { при всех } \Psi \in \mathbf{S}\right\} .
$$

Если в разделе 2 равенство $\left\langle X[\Phi]^{2}\right\rangle^{\mathrm{cl}}=0$ было равносильно равенству $X[\Phi]=0$, то теперь мы полагаем, что

$$
X[\Phi]=0 \text { в точности тогда, когда } \Phi \in \mathbf{S}^{\perp} .
$$

Определим факторпространство $\chi:=\mathbf{S} / \mathbf{S}^{\perp}$ с естественной проекцией $x: \Phi \mapsto x[\Phi] \in \chi$. Так как согласно (73) левая часть (71) зависит от $\Phi$ через $u:=x[\Phi]$ и от $\Psi$ через $\nu:=x[\Psi]$, то для левой части (71) мы будем использовать альтернативную запись $\widetilde{C}(\lambda \mid u, \nu)$. По той же причине форму (69) можно опустить на $\chi$. В результате $\chi$ есть вещественное пространство с невырожденным индефинитным скалярным произведением:

$$
(u \mid \nu)^{\mathrm{cl}} \equiv(x[\Phi] \mid x[\Psi])^{\mathrm{cl}}:=\beta \cdot\langle X[\Phi] \cdot X[\Psi]\rangle^{\mathrm{cl}}, \quad \Phi, \Psi \in \mathbf{S}, \quad u=x[\Phi], \quad \nu=x[\Psi] .
$$

Как и в разделе $2, X[\Phi]$ и $x[\Phi]$ имеют разный смысл: $X[\Phi]$ - это динамическая переменная, а $x[\Phi]$ - вектор из $\chi$. В соответствии с $(73) X[\Phi]$ зависит от $\Phi$ через 
$u=\chi[\Phi]$, и для $X[\Phi]$ мы будем использовать альтернативное обозначение $X[u]$. По аналогии с разделом 2 векторная обобщенная функция $x(t)$ определяется формулой $\int x(t) l \cdot \varphi(t) d t=x[\varphi \otimes l]$.

Правая часть (63) зависит от $s, t$ через разность $s-t$, поэтому в $\chi$ действует линейное представление $U(\tau)$ эволюционной группы $\mathbb{R}:$

$$
u=\chi[\Phi] \mapsto U(\tau) u:=\chi\left[\Phi_{\tau}\right], \quad(U(\tau) \chi[\Phi])(s ; l)=\chi[\Phi](s+\tau ; l),
$$

где, как и в (15), (16),

$$
\Phi_{\tau}:=\sum_{j}\left(\varphi_{j} \otimes l_{j}\right)_{\tau}=\sum_{j} \varphi_{j, \tau} \otimes l_{j}, \quad \varphi_{\tau}(t):=\varphi(t-\tau),
$$

так что $\Phi_{\tau} \in \mathbf{S}^{\perp}$ при $\Phi \in \mathbf{S}^{\perp}$. Формулы

$$
{ }^{\tau} X[u]:=X[U(\tau) u], \quad \int{ }^{\tau} X(t) l \cdot \varphi(t) d t:=\int X(t) l \cdot \varphi_{\tau}(t) d t
$$

распространяют действие $\mathbb{R}$ на динамические переменные. В частности,

$$
X(t) \mapsto{ }^{\tau} X(t)=X(t+\tau) .
$$

Генератор представления $U(\tau)$ в $\chi$ есть

$$
D:=\left.\partial_{\tau} U(\tau)\right|_{\tau=0}
$$

Очевидно,

$$
D x[\Phi]=x[\partial \Phi], \quad \text { где } \quad \partial \Phi:=\left.\partial_{\tau} \Phi_{\tau}\right|_{\tau=0} .
$$

Для дальнейшего заметим, что в силу (63), (75) при любых $u, \nu \in \chi(u \mid U(\tau) \nu)^{\mathrm{cl}}$ есть полиномиально ограниченная функция от $\tau$ класса $\mathrm{C}^{\infty}$ (таковы же и производные по $\tau$ ). Ее преобразование Фурье как обобщенная функция по $\tau$ выражается через $\widetilde{C}\left(\lambda ; l_{1}, l_{2}\right)$ по формуле (см. (71))

$$
F_{\tau \rightarrow \lambda}\left[(u \mid U(\tau) \nu)^{\mathrm{cl}}\right]=\widetilde{C}(\lambda \mid u ; \nu) .
$$

3.4. Условие отсутствия "нулевых мод". Как и в разделе 2, мы здесь исключаем возможность "нулевых мод", однако формулируем это иначе. Теперь мы предполагаем выполненным следующее условие (где используются обозначения $\mathrm{M}(\mathbb{R})$ и $\mathrm{M}_{0}(\mathbb{R})$ из соотношений $\left.(25),(26)\right)$.

УСловиЕ 1 (условие отсутствия "нулевых мод"). Если при некоторых $u, \nu \in \chi$ обобщенная функиия (80) такова, что выражение $\widetilde{C}(\lambda \mid u, \nu) \cdot d \lambda / 2 \pi$ является комплексной борелевской мерой на $\mathbb{R}$, то ее вклад в точке $\lambda=0$ равен нулю, т.е. из соотношения $\widetilde{C}(\lambda \mid u, \nu) \cdot d \lambda / 2 \pi \in \mathrm{M}(\mathbb{R})$ следует, что $\widetilde{C}(\lambda \mid u, \nu) \cdot d \lambda / 2 \pi \in \mathrm{M}_{0}(\mathbb{R})$.

СлЕДСтвиЕ 1. Линеал $\chi_{\mathrm{st}}$ стационарных векторов в $\chi$, определяемый как

$$
\chi_{\text {st }}:=\{u \in \chi: U(\tau) u=u \quad \text { при всех } \quad \tau \in \mathbb{R}\}=\{u \in \chi: D u=0\},
$$

равен нулю: $\chi_{\mathrm{st}}=\{0\}$. 
Доказательство. Пусть $u \in \chi, D u=0$. Докажем, что $u=0$.

При всех $\nu \in \chi$ имеем $\widetilde{C}(\lambda \mid D u, \nu)=0$, откуда $\lambda \cdot \widetilde{C}(\lambda \mid u, \nu)=0$, так что обобщенная функция $\widetilde{C}(\lambda \mid u, \nu)$ пропорциональна $\delta(\lambda)$. Согласно условию 1 имеем $\widetilde{C}(\lambda \mid u, \nu)=0$, следовательно,

$$
(u \mid \nu)^{\mathrm{cl}} \equiv \int \widetilde{C}(\lambda \mid u, \nu) \frac{d \lambda}{2 \pi}=0 .
$$

В силу невырожденности скалярного произведения в $\chi$ отсюда следует, что $u=0$.

СледСтвИЕ 2. Пусть при некоторых $u, \nu \in \chi$ существует обобщенная функция $h(\lambda)$ nо $\lambda$ класса $S^{\prime}(\mathbb{R})$ такал, что $h(\lambda) d \lambda / 2 \pi \in \mathrm{M}_{0}(\mathbb{R})$ u $\widetilde{C}(\lambda \mid u, \nu)=h(\lambda)$ nри $\lambda \neq 0$. Тогда $\widetilde{C}(\lambda \mid u, \nu)=h(\lambda)$ при $\lambda \in \mathbb{R}$.

ДокАЗАТЕЛЬСТво. По условию разность $\widetilde{C}(\lambda \mid u, \nu)-h(\lambda)$ есть обобщенная функция с носителем в нуле. Следовательно,

$$
\widetilde{C}(\lambda \mid u, \nu)=h(\lambda)+\sum_{k=0}^{m} a_{k} \delta^{(k)}(\lambda),
$$

где $m \geqslant 0$ - целое число, $a_{k}, k=0,1, \ldots, m,-$ комплексные числа. Достаточно доказать, что $a_{k}=0$ при всех $k$. Из (79) следует

$$
\widetilde{C}\left(\lambda \mid u, D^{m} \nu\right) \equiv(i \lambda)^{m} \widetilde{C}(\lambda \mid u, \nu)=(i \lambda)^{m} h(\lambda)+(-i)^{m} m ! a_{m} \delta(\lambda),
$$

так что $\widetilde{C}\left(\lambda \mid u, D^{m} \nu\right) d \lambda / 2 \pi \in \mathrm{M}(\mathbb{R})$. В силу условия $1 \widetilde{C}\left(\lambda \mid u, D^{m} \nu\right) d \lambda / 2 \pi \in \mathrm{M}_{0}(\mathbb{R})$, так что $a_{m}=0$ в (83). С помощью индукции по $k$ таким же образом доказывается, что $a_{m-k}=0$ при $k \leqslant m$.

3.5. Условие конечности ранга индефинитности. Следующие условия 2, 3 позволяют ввести в $\chi$ норму, благодаря которой $\chi$ становится плотным линеалом пространства Понтрягина с индефинитной метрикой. Условие 4 (см. п. 3.6) связывает индефинитность метрики с инфракрасными особенностями двухточечной функции.

УСловие 2. В пространстве х выделен (так называемый неотрицательный) линеал $\chi_{0}$, на котором скалярное произведение (74) неотрицательно определено. Линеал $\chi_{0}$ инвариантен относительно действия эволючионной группь $\mathbb{R}$ в $\chi$ и имеет конечную коразмерность $n \geqslant 1$ относительно $\chi\left(\operatorname{dim}\left(\chi / \chi_{0}\right)=n\right)$.

Формула

$$
\left(\|u\|^{\mathrm{cl}}\right)^{2}:=(u \mid u)^{\mathrm{cl}}, \quad u \in \chi_{0},
$$

определяет преднорму $\|u\|^{\mathrm{cl}}$ на $\chi_{0}$, так что

$$
\chi_{0}^{\perp}:=\left\{u \in \chi_{0}:\|u\|^{\mathrm{cl}}=0\right\}
$$

есть линеал нулевой преднормы в $\chi_{0}$. Из неравенства Коши-Буняковского

$$
\left|(u \mid \nu)^{\mathrm{cl}}\right| \leqslant\|u\|^{\mathrm{cl}} \cdot\|\nu\|^{\mathrm{cl}}, \quad u, \nu \in \chi_{0},
$$

следует

$$
(u \mid \nu)^{\mathrm{cl}}=0, \quad u \in \chi_{0}^{\perp}, \quad \nu \in \chi_{0} .
$$


УСловиЕ 3. Если для вектора $u \in \chi$ существует число $c \equiv c(u)$ такое, что

$$
\left|(u \mid \nu)^{\mathrm{cl}}\right| \leqslant c\|\nu\|^{\mathrm{cl}} \quad \text { дляв всех } \quad \nu \in \chi_{0},
$$

mo $u \in \chi_{0}$.

$\chi_{0}$ является максимальным неотрицательным линеалом в $\chi$, так как если существует неотрицательный линеал $\chi^{+}$в $\chi$, содержащий $\chi_{0}$, то неравенство (85) выполняется при $u \in \chi^{+}, \nu \in \chi_{0}$, что согласно условию 3 означает, что $\chi^{+}=\chi_{0}$.

Факторпространство $\chi_{0} / \chi_{0}^{\perp}$ есть предгильбертово пространство, в котором действует изометрическое представление эволюционной группы $\mathbb{R}$, поэтому здесь применимы соотношения (25). С учетом (80) они принимают вид

$$
\widetilde{C}(\lambda \mid u, u) \frac{d \lambda}{2 \pi} \in \mathrm{M}^{+}(\mathbb{R}), \quad \widetilde{C}(\lambda \mid u, \nu) \frac{d \lambda}{2 \pi} \in \mathrm{M}(\mathbb{R}), \quad u, \nu \in \chi_{0} .
$$

Условие 1, очевидно, усиливает эти соотношения:

$$
\widetilde{C}(\lambda \mid u, u) \frac{d \lambda}{2 \pi} \in \mathrm{M}_{0}^{+}(\mathbb{R}), \quad \widetilde{C}(\lambda \mid u, \nu) \frac{d \lambda}{2 \pi} \in \mathrm{M}_{0}(\mathbb{R}), \quad u, \nu \in \chi_{0} .
$$

Факторпространство $\mathcal{Z}:=\chi / \chi_{0}$ с естественной проекцией $Z: \chi \rightarrow \mathcal{Z}$ (задающей линейное расслоение) мы назовем пространством зарядов рассматриваемой системы. Это вещественное векторное пространство размерности $n$. Оно расслаивает $\chi$ на аффинные подпространства

$$
\chi_{z}:=\{u \in \chi: Z u=z\}
$$

с фиксированным значением $z$ заряда. Вещественное векторное пространство $G$, сопряженное к $\mathcal{Z}$, назовем калибровочной группой (абелевой первого рода) системы. Элемент $g \in G$ есть линейная форма на $\mathcal{Z}$, и $g \cdot z$ есть значение $g$ на векторе $z \in \mathcal{Z}$.

\section{6. Связь индефинитности с инфракрасными особенностями двухто-} чечной функции. На действие эволюционной группы $U(\tau)$ мы наложим "техническое" условие 4, которое выполняется во многих физически интересных моделях, в частности для фрактальных броуновских движений (67).

УСловиЕ 4. Заряд инвариантен относительно временных трансляиий:

$$
Z U(\tau) u=Z u, \quad u \in \chi .
$$

Это условие может быть записано в следующих эквивалентных формах:

$$
\begin{gathered}
Z D u=0, \quad u \in \chi, \\
\tau \cdot D \chi \subset \chi_{0}
\end{gathered}
$$

(здесь множитель $\tau$ присутствует по соображениям размерности). Из условия 4 выводится

СлЕДСтвиЕ 3. Преднорма $\|u\|^{\mathrm{cl}}$ на $\chi_{0}$ есть норма, m.е. $\chi_{0}^{\perp}=\{0\}$. 
ДокАЗАТЕЛЬСтво. Пусть $u \in \chi_{0}$ и $\|u\|^{\mathrm{cl}}=0$. Нужно доказать, что $u=0$. Согласно (93) $\tau \cdot D \nu \in \chi_{0}$ при всех $\nu \in \chi$, и из равенства $(86)$ имеем $0=(u \mid D \nu)^{\mathrm{cl}} \equiv$ $-(D u \mid \nu)^{\mathrm{cl}}$. В силу невырожденности скалярного произведения в $\chi$ отсюда следует $D u=0$. Теперь из следствия 1 вытекает доказываемое равенство $u=0$.

СлЕДСТВиЕ 4. Пусть $u=x[\Phi] \equiv x\left[\sum_{j} \varphi_{j} \otimes l_{j}\right] \in \chi$, тогда

$$
Z u=\sum_{j} \tilde{\varphi}_{j}(0) \cdot \zeta l_{j}
$$

где $\zeta: L \rightarrow \mathcal{Z}$ - линейное отображение $L$ на $\mathcal{Z} . B$ частности, $u \in \chi_{0}$ в точности тогда, когда правая часть (94) равна нулю.

ДокАзАТЕЛЬСтво. Формула (94) означает, что $Z$ u зависит линейно только от вектора $\sum_{j} \tilde{\varphi}_{j}(0) \cdot l_{j}$. Достаточно доказать, что если этот вектор равен нулю, то $Z u=0$. Пусть $l_{j}=\sum_{k} e_{k} a_{k j}-$ разложение векторов $l_{j}$ по линейно независимым векторам $e_{k}$. Тогда $\sum_{j} a_{k j} \tilde{\varphi}_{j}(0)=0$, поэтому $\sum_{j} a_{k j} \varphi_{j}(t)=\partial_{t} \psi_{k}(t)$, где $\psi_{k} \in \mathrm{S}_{\mathrm{r}}(\mathbb{R})$. Положим $\Psi=\sum_{k} \psi_{k} \otimes e_{k}$, тогда $u=x[\Phi]=D x[\Psi]$, и в силу (92) $Z u=0$. Это доказывает существование линейного отображения $\zeta: L \rightarrow \mathcal{Z}$. То, что оно сюръективно, следует из сюръективности $Z$.

Согласно следствию $3 \chi_{0}$ есть предгильбертово пространство с нормой $\|u\|^{\mathrm{cl}}$. Обозначим через $H_{0}^{\mathrm{cl}}$ вещественное гильбертово пространство - пополнение $\chi_{0}$. Скалярное произведение в $H_{0}^{\mathrm{cl}}$ по-прежнему запишем в виде $(u \mid \nu)^{\mathrm{cl}}$. Изометрическое представление в $\chi_{0}$ группы $\mathbb{R}$ (сужение $U(\tau)$ на $\left.\chi_{0}\right)$ продолжается по непрерывности до изометрического представления $U_{0}(\tau)$ в $H_{0}^{\text {cl }}$ с генератором $D_{0}$ :

$$
D_{0}:=\Omega J=J \Omega, \quad \Omega>0, \quad J^{*}=-J, \quad J^{2}=-1 .
$$

В этой ситуации справедливы соотношения, аналогичные (25), которые с учетом условия 1 и обозначения (80) можно записать в форме, аналогичной (26):

$$
\begin{array}{ll}
F_{\tau \rightarrow \lambda}\left[\left(u \mid U_{0}(\tau) u\right)^{\mathrm{cl}}\right] \frac{d \lambda}{2 \pi} \in \mathrm{M}_{0}^{+}(\mathbb{R}), & u \in \chi_{0}, \\
F_{\tau \rightarrow \lambda}\left[\left(u \mid U_{0}(\tau) \nu\right)^{\mathrm{cl}}\right] \frac{d \lambda}{2 \pi} \in \mathrm{M}_{0}(\mathbb{R}), & u, \nu \in \chi_{0} .
\end{array}
$$

СледСТВиЕ 5. Для представления $U_{0}(\tau)$ в $H_{0}^{\mathrm{cl}}$ условие отсутствия "нулевых мод" выполнено в борме (21), т.е.

$$
\left\{u \in H_{0}^{\mathrm{cl}}: U_{0}(\tau) u=u \text { nрu всех } \tau \in \mathbb{R}\right\} \equiv D_{0}^{-1}\{0\}=\{0\} .
$$

ДокАЗАтельство. Достаточно проверить, что проектор $\mathbf{P}_{\mathrm{st}}$ в $H_{0}^{\mathrm{cl}}$ на линеал стационарных векторов равен нулю. В силу (96) при $u \in \chi_{0}$ имеем

$$
\left(u \mid \mathbf{P}_{\mathrm{st}} u\right)^{\mathrm{cl}} \equiv \int_{\lambda=0} F_{\tau \rightarrow \lambda}\left[\left(u \mid U_{0}(\tau) u\right)^{\mathrm{cl}}\right] \frac{d \lambda}{2 \pi}=\int_{\lambda=0} \widetilde{C}(\lambda \mid u, u) \frac{d \lambda}{2 \pi}=0 .
$$

В силу плотности $\chi_{0}$ в $H_{0}^{\mathrm{cl}}$ отсюда вытекает, что $\mathbf{P}_{\mathrm{st}}=0$.

Условие 4 означает, что индефинитность скалярного произведения в $\chi$ обусловлена инфракрасными сингулярностями двухточечной функции. Оно же ограничивает степень допустимой сингулярности. Действительно, согласно (79), (80)

$$
\widetilde{C}(\lambda \mid D u, D \nu)=\lambda^{2} \widetilde{C}(\lambda \mid u, \nu),
$$


так что выражения

$$
d \mu(\lambda \mid u, \nu):=\lambda^{2} \widetilde{C}(\lambda \mid u, \nu) \frac{d \lambda}{2 \pi} \in \mathrm{M}(\mathbb{R}), \quad u, \nu \in \chi
$$

в силу (93), (96) являются конечными комплексными борелевскими мерами на $\mathbb{R}$ с нулевым вкладом в точке $\lambda=0$ :

$$
d \mu(\lambda \mid u, u) \in \mathrm{M}_{0}^{+}(\mathbb{R}), \quad d \mu(\lambda \mid u, \nu) \in \mathrm{M}_{0}^{+}(\mathbb{R}), \quad u, \nu \in \chi .
$$

Соотношения (98), (99) означают, что степень сингулярности $\widetilde{C}(\lambda \mid u, \nu)$ может превосходить степень сингулярности борелевской меры на $\mathbb{R}$ не более, чем на 2.

Кроме того, условие 4 позволяет дать непосредственную характеризацию $\chi_{0}$ через двухточечную функцию.

СлЕДСТВиЕ 6. Линеал $\chi_{0}$ характеризуется условием

$$
\chi_{0}=\left\{u \in \chi: \int \lambda^{-2} d \mu(\lambda \mid u, u) \frac{d \lambda}{2 \pi}<+\infty\right\} .
$$

ДокАЗАтЕЛьство. Обозначим правую часть (100) через $\chi^{+}$. Требуется доказать, что $\chi^{+}=\chi_{0}$. Заметим, что $\chi^{+}$есть линеал в $\chi$. Это следует из того, что $\chi^{+}$ есть конус (с вершиной в нуле), и из тождества

$$
d \mu(\lambda \mid u+\nu, u+\nu)+d \mu(\lambda \mid u-\nu, u-\nu)=2 d \mu(\lambda \mid u, u)+2 d \mu(\lambda \mid \nu, \nu), \quad u, \nu \in \chi .
$$

Докажем, что $(u \mid u)^{\mathrm{cl}} \geqslant 0$ при $u \in \chi^{+}$. Пусть $u \in \chi^{+}$. Из определения $\chi^{+}$следует, что существует обобщенная функция $h(\lambda)$ по $\lambda$ класса $S^{\prime}(\mathbb{R})$ такая, что $h(\lambda) d \lambda / 2 \pi \in$ $\mathrm{M}_{0}(\mathbb{R})$ и $\lambda^{-2} d \mu(\lambda \mid u, u)=h(\lambda \mid u, u) d \lambda / 2 \pi$ при $\lambda \neq 0$. Тогда в силу (98) имеем $\widetilde{C}(\lambda \mid u, u)=h(\lambda)$ при $\lambda \neq 0$. Следствие 1 позволяет продолжить это равенство: $\widetilde{C}(\lambda \mid u, u)=h(\lambda)$ при $\lambda \in \mathbb{R}$. В результате имеем

$$
(u \mid u)^{\mathrm{cl}} \equiv \int \widetilde{C}(\lambda \mid u, u) \frac{d \lambda}{2 \pi}=\int h(\lambda) \frac{d \lambda}{2 \pi} \geqslant 0 .
$$

Итак, $\chi^{+}-$неотрицательный линеал в $\chi$. Пусть теперь $u \in \chi_{0}$. Тогда соотношение (98) позволяет привести интеграл $\int \lambda^{-2} d \mu(\lambda \mid u, u) d \lambda / 2 \pi$ к виду $\int \widetilde{C}(\lambda \mid u, u) d \lambda / 2 \pi$, где согласно (89) $\widetilde{C}(\lambda \mid u, u) d \lambda / 2 \pi \in \mathrm{M}_{0}^{+}(\mathbb{R})$. Таким образом, $\int \lambda^{-2} d \mu(\lambda \mid u, u) d \lambda / 2 \pi<$ $+\infty$ при $u \in \chi_{0}$, а это означает, что $\chi_{0} \subset \chi^{+}$. Так как $\chi_{0}-$ максимальный неотрицательный линеал в $\chi$, то в результате получаем, что $\chi^{+}=\chi_{0}$.

ЗАмЕчАниЕ 5. На $\chi$ определена структура вещественного предгильбертова пространства. Можно выбрать набор векторов $\left\{b_{\alpha}\right\}_{\alpha=1, \ldots, n}$ в $\chi$ таких, что $\left\{Z b_{\alpha}\right\}-$ базис в $\mathcal{Z}$ и $\left(b_{\alpha} \mid b_{\beta}\right)^{\text {cl }}=0$. Произвольный элемент $u \in \chi$ однозначно представляется в виде $u \equiv u_{0}+\sum_{\alpha} z_{\alpha} b_{\alpha}$, где $u_{0} \in \chi_{0}, z_{\alpha} \in \mathbb{R}, Z u=\sum_{\alpha} z_{\alpha} \cdot Z b_{\alpha}$. Зададим норму $|u|$ элемента $u$ формулой

$$
|u|^{2} \equiv\left|u_{0}+\sum_{\alpha} z_{\alpha} b_{\alpha}\right|^{2}:=\left(\left\|u_{0}\right\|^{\mathrm{cl}}\right)^{2}+\sum_{\alpha}\left(z_{\alpha}\right)^{2}+\sum_{\alpha}\left(\left(b_{\alpha} \mid u\right)^{\mathrm{cl}}\right)^{2} .
$$


Обозначим через $H^{\mathrm{cl}}$ пополнение $\chi$ по этой норме. Топология $H^{\mathrm{cl}}$ совпадает с топологией прямой суммы $\bar{\chi}_{0} \oplus \mathcal{Z}$, где $\bar{\chi}_{0} \approx H_{0}^{\mathrm{cl}} \oplus G$ - замыкание $\chi_{0}$ в $H^{\mathrm{cl}}$. Индефинитное скалярное произведение $(74)$ в $\chi$ продолжается по непрерывности на все $H^{\mathrm{cl}}$. При этом $H^{\mathrm{cl}}$ становится вещественным пространством Понтрягина с индексом индефинитности $n([26]$, гл. $1, \S 9)$, а $\chi$ - плотным линеалом в $H^{\mathrm{cl}}$. Действие $U(\tau)$ эволюционной группы $\mathbb{R}$ в $\chi$ продолжается линейными непрерывными операторами в $H^{\mathrm{cl}}$, которые по-прежнему обозначаются как $U(\tau)$ и которые непрерывны по $\tau$ в сильной операторной топологии. Область определения генератора $D$ представления $U(\tau)$ в $H^{\mathrm{cl}}$ содержит $\chi$.

Данное определение $H^{c l}$ позволяет сделать вывод, что по формально заданной двухточечной функции, удовлетворяющей условиям 1-4, можно реконструировать векторный виртуальный процесс, определенный в п. 3.2.

3.7. Фазовое пространство полной системы. Определим билинейную кососимметрическую форму на $\chi$ :

$$
\sigma(u, \nu):=-(D u \mid \nu)^{\mathrm{cl}}=(u \mid D \nu)^{\mathrm{cl}}, \quad u, \nu \in \chi .
$$

Согласно следствию 1 форма $\sigma$ невырожденна, т. е. является симплектической. Симплектическое пространство $\chi$ интепретируется как фазовое пространство полной системы.

Покажем, что полная система в классическом режиме представляет собой детерминированную гамильтонову систему, в которой роль канонических переменных играют компоненты векторной динамической переменной $Y^{\mathrm{cl}}(t)$ над симплектическим пространством $\chi$. А именно, каждому вектору $u \in \chi$ сопоставляется вещественная динамическая переменная $Y^{\mathrm{cl}}(t) u$, линейно зависящая от $u^{8)}$. Для одновременных скобок Пуассона переменных $Y^{\mathrm{cl}}(t) u$ постулируется выражение

$$
\left\{Y^{\mathrm{cl}}(t) u, Y^{\mathrm{cl}}(t) \nu\right\}:=\sigma(u, \nu), \quad u, \nu \in \chi .
$$

Пусть $\left\{e_{a}\right\}_{a \in A}-$ базис в $H^{\mathrm{cl}}$ в топологии вещественного гильбертова пространства. По аналогии с (30) гамильтониан полной системы задается формальным выражением

$$
\mathrm{H} \equiv \mathrm{H}\left(Y^{\mathrm{cl}}(t)\right):=\frac{1}{2} \sum_{a, b} Y^{\mathrm{cl}}(t) e_{a} \cdot\left(\mathrm{M}^{-1}\right)^{a b} \cdot Y^{\mathrm{cl}}(t) e_{b},
$$

где $\mathrm{M}^{-1}$ - матрица, обратная к "метрическому тензору" $\mathrm{M} \equiv\left(\mathrm{M}_{a b}\right):=\left(\left(e_{a} \mid e_{b}\right)^{\mathrm{cl}}\right)$ в $H^{\mathrm{cl}}$. Отсюда получаем гамильтоновы уравнения движения

$$
\partial_{t} Y^{\mathrm{cl}}(t) u=Y(t) D u, \quad u \in \chi
$$

Действительно, пусть $u, \nu \in \chi$. Учитывая соотношение $\sum_{a, b} e_{a} \cdot\left(\mathrm{M}^{-1}\right)^{a b} \cdot\left(e_{b} \mid u\right)^{\mathrm{cl}} \equiv u$, имеем

$$
\begin{aligned}
\partial_{t} Y^{\mathrm{cl}}(t) u & =\left\{\mathrm{H}, Y^{\mathrm{cl}}(t) u\right\}=\sum_{a, b} Y^{\mathrm{cl}}(t) e_{a} \cdot\left(\mathrm{M}^{-1}\right)^{a b} \cdot \sigma\left(e_{b}, u\right)= \\
& =Y^{\mathrm{cl}}(t) \sum e_{a} \cdot\left(\mathrm{M}^{-1}\right)^{a b} \cdot\left(e_{b} \mid D u\right)^{\mathrm{cl}}=Y^{\mathrm{cl}}(t) D u
\end{aligned}
$$

8) Можно считать, что $Y^{\mathrm{cl}}(t) u$ определено на векторах $u$ из $H^{\mathrm{cl}}$ - пополнения $\chi$ по норме (101). Однако нигде, кроме формул (104), (106), это не используется в дальнейшем. 
Решением уравнения (105) является формула, аналогичная (31). Это означает, что $U(t)$ - гамильтонов фазовый поток с гамильтонианом Н. Из (103), (31) следует формула для двухвременных скобок Пуассона - аналог (32):

$$
\begin{aligned}
\left\{Y^{\mathrm{cl}}(s) u, Y^{\mathrm{cl}}(t) \nu\right\} & =\sigma(u, U(t-s) \nu)=(u \mid \mathcal{D}(s-t) \nu)^{\mathrm{cl}}, \quad u, \nu \in \chi, \\
\mathcal{D}(s-t) & :=D U(t-s)=-\partial_{s} U(t-s) .
\end{aligned}
$$

3.8. Алгебра KКС полной системы. При квантовании скобкам Пуассона (103) при $t=0$ соответствуют ККС полной системы

$$
\left[Y^{\mathrm{qu}}(0) u, Y^{\mathrm{qu}}(0) \nu\right]=-i \hbar \sigma(u, \nu), \quad u, \nu \in \chi .
$$

Поскольку мы имеем дело с линейной гамильтоновой системой, в квантовой области выполняются соотношение (31) и квантовый аналог формулы (107). С помощью унитарных элементов $\mathcal{E}_{u}$ при $u \in \chi \mathrm{KKC}$ записываются в форме Вейля

$$
\mathcal{E}_{u} \mathcal{E}_{\nu}=e^{(i \hbar / 2) \sigma(u, \nu)} \mathcal{E}_{u+\nu}, \quad u, \nu \in \chi .
$$

$\mathrm{C}$ системой $\mathrm{KKC}$ ассоциируется $C^{*}$-алгебра $\mathrm{KKC}$ [27] - индуктивный предел алгебр фон Неймана $\mathcal{A}_{\mathrm{vN}}(M)$, сопоставляемых конечномерным симплектическим линеалам $M \subset \chi$ и порождаемых операторами $\pi_{\mathrm{S}}\left(\mathcal{E}_{u}\right)$ шредингеровского представления ККС над $M$. Несколько сузив эту алгебру, будем считать (в духе [28]), что $*$-алгебра ККС $\mathcal{A}^{\mathrm{qu}}(\chi)$ полной системы есть индуктивный предел $*$-подалгебр $\mathcal{A}^{\mathrm{qu}}(M)$, состоящих из формальных интегралов

$$
A=\int_{M} \mathcal{E}_{u} d \rho(u)
$$

с нормой $\|A\|=\left\|\int_{M} \pi_{\mathrm{S}}\left(\mathcal{E}_{u}\right) d \rho(u)\right\|$, где $M$ - конечномерный симплектический линеал в $\chi$, а $\rho$ - произвольная комплексная борелева мера на $M$ с компактным носителем. При переходе к регулярному представлению $\mathrm{KKC} \pi$ в комплексном гильбертовом пространстве формальным интегралам (111) соответствуют обычные интегралы $\pi(A)=\int_{M} \pi\left(\mathcal{E}_{u}\right) d \rho(u)$. Регулярность $x$ означает, что $\pi\left(\mathcal{E}_{t u}\right)$ непрерывно зависит от $t \in \mathbb{R}$ в слабой операторной топологии, что позволяет представить $\pi\left(\mathcal{E}_{u}\right)$ в экспоненциальном виде $\pi\left(\mathcal{E}_{u}\right)=e^{i Y^{\text {qu }}(0) u}$. Элементам $\mathcal{E}_{\nu}$ соответствует единичная мера $\rho$, сосредоточенная в точке $u=\nu$. Эволюционная группа $\mathbb{R}$ действует в $\mathcal{A}^{\text {qu }}(\chi)$ *-автоморфизмами $\delta_{\tau}$, которые сводятся к замене $\mathcal{E}_{u}$ в $(111)$ на

$$
\delta_{\tau} \mathcal{E}_{u}:=\mathcal{E}_{U_{(\tau) u}} .
$$

Попутно определим абелеву *-алгебру $\mathcal{A}^{\mathrm{cl}}(\chi)$ полной системы в классическом режиме. Она является индуктивным пределом *-подалгебр $\mathcal{A}^{\mathrm{cl}}(M)$, состоящих из формальных интегралов (111) с нормой

$$
\|A\|=\sup _{p}\left|\int_{M} e^{i(p, u)} d \rho(u)\right|,
$$

где $p$ - вектор сопряженного пространства к $M$. Унитарные элементы $\mathcal{E}_{u}$ удовлетворяют соотношениям

$$
\mathcal{E}_{u} \mathcal{E}_{\nu}=\mathcal{E}_{u+\nu}, \quad\left\{\mathcal{E}_{u}, \mathcal{E}_{\nu}\right\}=-\sigma(u, \nu) \cdot \mathcal{E}_{u+\nu}
$$


и в регулярном представлении $\pi\left(\mathcal{E}_{u}\right)=e^{i Y^{\mathrm{cl}}(0) u}$. Эволюционная группа действует на $\mathcal{A}^{\mathrm{cl}}(\chi)$ по аналогии с $(112)$.

Нас интересуют температурные представления, в первую очередь, квантовой *-алгебры $\mathcal{A}^{\mathrm{qu}}(\chi)$. Элементу $g$ сопоставляется неоднородное линейное каноническое преобразование динамических переменных

$$
Y^{\mathrm{qu}}(0) u \rightarrow Y^{\mathrm{qu}}(0) u+g \cdot Z u
$$

Тем самым группа $G$ действует *-автоморфизмами $\gamma_{g}$ *-алгебры $\mathcal{A}^{\text {qu }}(\chi)$ (а также $\left.\mathcal{A}^{\mathrm{cl}}(\chi)\right)$, которые сводятся к замене $\mathcal{E}_{u}$ в $(111)$ на

$$
\gamma_{g} \mathcal{E}_{u}:=e^{i g \cdot Z u} \mathcal{E}_{u}
$$

Обозначим через $\mathcal{A}^{\mathrm{qu}}\left(\chi_{0}\right)$ *-подалгебру в $\mathcal{A}^{\mathrm{qu}}(\chi)$, состоящую из элементов $A$ вида (111) с носителями $\rho$ на пересечении $M$ с $\chi_{0}$. Мы назовем ее подалгеброй калибровочно-инвариантных элементов из $\mathcal{A}^{\text {qu }}(\chi)$. Аналогично определяется $\mathcal{A}^{\mathrm{cl}}\left(\chi_{0}\right) \subset$ $\mathcal{A}^{\mathrm{cl}}(\chi)$.

Выделим в $\mathcal{A}^{\mathrm{qu}}(\chi)$ подмножество $\mathcal{J}^{\mathrm{qu}}$, состоящее из элементов $A$ вида $(111)$, где мера $d \rho$ такова, что для любой комплексной непрерывной функции $h$ по $u$ с компактным носителем на $M$ обобщенная функция $\hat{h}(z)$ из $S^{\prime}(\mathcal{Z})$ по $z$

$$
\hat{h}(z)=\int_{M}(2 \pi)^{n} \delta(z-Z u) h(u) d \rho(u)
$$

непрерывна, так что имеет смысл сужение

$$
\hat{h}(0)=\int_{M}(2 \pi)^{n} \delta(Z u) h(u) d \rho(u) .
$$

Такой элемент $A$ мы назовем $\mathcal{Z}$-непрерывным. Примером $\mathcal{Z}$-непрерывного элемента служит элемент (111) при $Z M \supset \mathcal{Z}$ и $d \rho(u)=k(u) d r(u)$, где $k(u)$ - комплексная непрерывная функция с компактным носителем на $M$, а $d r(u)$ - мера Лебега на $M$. Очевидно, что если $A-\mathcal{Z}$-непрерывный элемент, то таков же элемент $\mathcal{E}_{u} A \mathcal{E}_{\nu}$ при любых $u, \nu \in \chi$. Более общее утверждение получается, если рассмотреть произведение $A=A_{1} A_{2}$ элементов вида (111) с мерами $\rho_{1}$ и $\rho_{2}$ на $M_{1}$ и $M_{2}: A$ имеет вид (111) с мерой $\rho$ на $M \supset M_{1}+M_{2}$, называемой сверткой с кручением мер $\rho_{1}, \rho_{2}$ :

$$
d \rho(u):=\left[\int_{M} \int_{M} \delta\left(u-u_{1}-u_{2}\right) e^{(i \hbar / 2) \sigma\left(u_{1}, u_{2}\right)} d \rho_{1}\left(u_{1}\right) d \rho_{2}\left(u_{2}\right)\right] d r(u) .
$$

Отсюда можно вывести, что $\mathcal{J}^{\text {qu }}$ есть $*$-идеал в $\mathcal{A}^{\text {qu}}(\chi)$. Аналогично определяется идеал $\mathcal{J}^{\mathrm{cl}} \subset \mathcal{A}^{\mathrm{cl}}(\chi)$, состоящий из $\mathcal{Z}$-непрерывных элементов.

На $\mathcal{Z}$-непрерывных элементах $A \in \mathcal{J}^{\text {qu }}$ определено обобщенное условное ожидание $m^{\text {qu }}$ как интеграл по калибровочной группе $G$ (в слабой операторной топологии представления $\pi_{\mathbf{S}}$ подалгебр $\left.\mathcal{A}_{\mathrm{vN}}(M)\right)$ :

$$
m^{\mathrm{qu}}(A):=\int_{M} \gamma_{g} A d g=\int_{M}(2 \pi)^{n} \delta(Z u) \mathcal{E}_{u} d \rho(u)
$$


Предполагается, что на $G$ и на $\mathcal{Z}$ как на сопряженных пространствах меры Лебега $d g$ и $d^{n} z$ определены согласованным образом. Оператор $m^{\text {qu }}$ переводит $\mathcal{Z}$-непрерывные элементы в калибровочно-инвариантные ${ }^{9)}$.

Аналогичным образом определено обобщенное условное ожидание $m^{\mathrm{cl}}: \mathcal{J}^{\mathrm{cl}} \rightarrow$ $\mathcal{A}^{\mathrm{cl}}\left(\chi_{0}\right)$.

\section{9. Гиббсовское представление подалгебры калибровочно-инвариант-} ных величин. Благодаря условию 2 из динамических переменных $Y^{\mathrm{cl}}(t) u$ при $u \in$ $\chi_{0}$ можно составить классический векторный гауссовский процесс $Y_{0}^{\mathrm{cl}}(t) u$ с нулевым средним и с двухточечной функцией

$$
\left\langle Y_{0}^{\mathrm{cl}}(s) u \cdot Y_{0}^{\mathrm{cl}}(t) \nu\right\rangle^{\mathrm{cl}}:=\left\langle Y^{\mathrm{cl}}(s) u \cdot Y^{\mathrm{cl}}(t) \nu\right\rangle^{\mathrm{cl}}=\beta^{-1}(u \mid U(t-s) \nu)^{\mathrm{cl}}, \quad u, \nu \in \chi_{0} .
$$

Он описывает эволюцию некоторой подсистемы. Таким образом, мы имеем стационарное гауссовское состояние $s_{0}^{\mathrm{cl}} *$-алгебры $\mathcal{A}^{\mathrm{cl}}\left(\chi_{0}\right)$ с характеристическим функционалом

$$
s_{0}^{\mathrm{cl}}\left(\mathcal{E}_{u}\right)=e^{-(u \mid u)^{\mathrm{cl}} / 2 \beta}, \quad u \in \chi_{0} .
$$

Результат раздела 2 позволяет перейти (по формулам (7), (48)) к квантовому векторному гауссовскому процессу $Y_{0}^{\mathrm{qu}}(t)$ над $\chi_{0}$ с двухточечной функцией

$$
\left\langle Y_{0}^{\mathrm{qu}}(s) u \cdot Y_{0}^{\mathrm{qu}}(t) \nu\right\rangle^{\mathrm{qu}}=\beta^{-1} \mathrm{~B}\left(i \beta \hbar \partial_{s}\right)(u \mid U(t-s) \nu)^{\mathrm{cl}}, \quad u, \nu \in \chi_{0} .
$$

Это равносильно заданию стационарного состояния $s_{0}^{\text {qu }}$ алгебры $\mathcal{A}^{\mathrm{qu}}\left(\chi_{0}\right)$ с характеристическим функционалом

$$
s_{0}^{\mathrm{qu}}\left(\mathcal{E}_{u}\right)=e^{-(u \mid \mathbf{Q} u)^{\mathrm{cl}} / 2 \beta}, \quad u \in \chi_{0},
$$

где в соответствии с формулой (55)

$$
\left\langle\left(Y_{0}^{\mathrm{qu}}(t) u\right)^{2}\right\rangle^{\mathrm{qu}}=\beta^{-1}(u \mid \mathbf{Q} u)^{\mathrm{cl}}, \quad u \in \chi_{0},
$$

a $\mathbf{Q}=(\beta \hbar \Omega / 2) \operatorname{cth}(\beta \hbar \Omega / 2)>1$ - положительный оператор типа (56) в $H_{0}^{\mathrm{cl}}$, а оператор $\Omega$ определен в (95). Введем также норму $\|u\|^{\text {qu }}$ в $\chi_{0}$ (которая, в отличие от $\|u\|^{\text {cl }}$, зависит от $\left.\beta\right)$ :

$$
\left(\|u\|^{\mathrm{qu}}\right)^{2}:=(u \mid \mathbf{Q} u)^{\mathrm{cl}}, \quad u \in \chi_{0} .
$$

Обозначим через $H_{0}^{\mathrm{qu}}$ пополнение $\chi_{0}$ по этой норме $\left(H_{0}^{\mathrm{qu}} \subset H_{0}^{\mathrm{cl}}\right)$.

Отметим, что в силу (102), (95), (122) симплектическая форма на $\chi_{0}$ в терминах $(u \mid \nu)^{\text {qu }}$ имеет вид

$$
\sigma(u, \nu)=\frac{2}{\beta \hbar}(u \mid J \omega \nu)^{\mathrm{qu}}, \quad \text { где } \quad \omega=\operatorname{th} \frac{\beta \hbar \Omega}{2}, \quad u, \nu \in \chi_{0} .
$$

Далее в однотипных формулах для классического и квантового режимов мы используем верхний символ $\sharp$, принимающий два значения: "cl" и "qu". Так, значение состояния $s_{0}^{\sharp}$ на элементе $A \in \mathcal{A}^{\sharp}\left(\chi_{0}\right)$ вида (111) есть

$$
s_{0}^{\sharp}(A)=\int_{M} s_{0}^{\sharp}\left(\mathcal{E}_{u}\right) d \rho(u) .
$$

${ }^{9)}$ Согласно [29] условное ожидание в $C^{*}$-алгебре с единицей есть линейное положительное отображение $m$ алгебры в себя со свойствами $m(1)=1, m(A \cdot m(B)) \equiv m(m(A) \cdot B) \equiv m(A) \cdot m(B)$. В отличие от этого, обобщенное условное ожидание определено не на всей алгебре, а на $*$-идеале. 
Известная процедура ГНС [22] позволяет построить представление Гиббса $\pi_{0}^{\sharp}$ подалгебры $\mathcal{A}^{\sharp}\left(\chi_{0}\right)$ в комплексном гильбертовом пространстве $\mathcal{H}_{0}^{\sharp}$ с циклическим вектором $|\mathrm{GIBBS}\rangle^{\sharp}$, так что векторы

$$
F_{0}^{\sharp} A:=\pi_{0}^{\sharp}(A)|\mathrm{GIBBS}\rangle^{\sharp}, \quad A \in \mathcal{A}^{\sharp}\left(\chi_{0}\right),
$$

образуют плотное множество в $\mathcal{H}_{0}^{\sharp}$ со скалярным произведением

$$
\left\langle F_{0}^{\sharp} A \mid F_{0}^{\sharp}(B)\right\rangle^{\sharp}:=s_{0}^{\sharp}\left(A^{*} B\right), \quad A, B \in \mathcal{A}^{\sharp}\left(\chi_{0}\right) .
$$

Эволюционная группа действует в $\mathcal{H}_{0}^{\sharp}$ унитарными операторами $\bigcup_{0}^{\sharp}(\tau)$ по правилу

$$
\mathrm{U}_{0}^{\sharp}(\tau) F_{0}^{\sharp} A=F_{0}^{\sharp}\left(\delta_{\tau} A\right), \quad A \in \mathcal{A}^{\sharp}\left(\chi_{0}\right), \quad \tau \in \mathbb{R} .
$$

Вектор $|\mathrm{GIBBS}\rangle^{\sharp}$ остается инвариантным.

3.10. Температурные представления алгебры полной (а также открытой) системы. Определим стационарное обобщенное состояние ${ }^{10)}$ системы $s^{\sharp}$ алгебры $\mathcal{A}^{\sharp}(\chi)$ как индуцированное состояние $s_{0}^{\sharp}$ подалгебры $\mathcal{A}^{\sharp}\left(\chi_{0}\right)$ калибровочно-инвариантных элементов посредством обобщенного условного ожидания $m^{\sharp}$. Его область определения есть $*$-идеал $\mathcal{J}^{\sharp}$ :

$$
s^{\sharp}(A):=s_{0}^{\sharp}\left(m^{\sharp}(A)\right), \quad A \in \mathcal{J}^{\sharp} .
$$

Характеристический функционал $s^{\sharp}$ имеет вид

$$
s^{\sharp}\left(\mathcal{E}_{u}\right)=(2 \pi)^{n} \delta(Z u) e^{-\left(\|u\|^{\sharp}\right)^{2} / 2}, \quad u \in \chi .
$$

Обобщенное состояние ненормируемо $\left(s^{\sharp}(1)=\infty\right)$. Если выражение (128) проинтегрировать по и с $\mathcal{Z}$-непрерывной мерой $d \rho$ (как в (111)), то в результате получится (127). Заметим, что из-за присутствия $\delta$-функции в выражении (128) существенна норма $\|u\|^{\sharp}$ только при $u \in \chi_{0}$; она была определена в (84), (122) только для таких $u$.

На элементах $A \in \mathcal{J}^{\sharp}$ выполняется неравенство $m^{\sharp}\left(A^{*} A\right) \geqslant 0$, так что выполнено условие положительности $s^{\sharp}\left(A^{*} A\right) \geqslant 0$ (при $\left.A \in \mathcal{J}^{\sharp}\right)$. Поэтому конструкция ГНС представления алгебры по ее состоянию легко переносится на обобщенное состояние. А именно, температурное представление $\pi^{\sharp} *$-алгебры $\mathcal{A}^{\sharp}(\chi)$ строится в комплексном гильбертовом пространстве $\mathcal{H}^{\sharp 11)}$. Плотное множество в $\mathcal{H}^{\sharp}$ образовано векторами $F^{\sharp} A$ (при $\left.A \in \mathcal{J}^{\sharp}\right)$, определяемыми двумя свойствами: они линейно зависят от $A$, а их скалярное произведение равно

$$
\left\langle F^{\sharp} A \mid F^{\sharp} B\right\rangle^{\sharp}:=s^{\sharp}\left(A^{*} B\right)=\left\langle\left.\mathrm{GIBBS}\right|^{\sharp} \pi_{0}^{\sharp}\left(m^{\sharp}\left(A^{*} B\right)\right) \mid \mathrm{GIBBS}\right\rangle^{\sharp}, \quad A, B \in \mathcal{J}^{\sharp} .
$$

Операторы $\pi^{\sharp}\left(\mathcal{E}_{u}\right)$ (при $\left.u \in \chi\right)$ конкретной бозонной системы над $\chi$ действуют по правилу

$$
\pi^{\sharp}\left(\mathcal{E}_{u}\right) F^{\sharp} A=F^{\sharp}\left(\mathcal{E}_{u} A\right), \quad A \in \mathcal{J}^{\sharp}, \quad u \in \chi,
$$

10) Обобщенные состояния - это так называемые "несобственные" состояния с бесконечной нормой, широко используемые в квантовой механике [30].

11) В отличие от $\mathcal{H}_{0}^{\sharp}$ пространство $\mathcal{H}^{\sharp}$ не обладает стационарным циклическим вектором; его роль играет некий стационарный циклический обобщенный вектор $|\mathrm{EQUI}\rangle$. В дальнейшем мы не пользуемся этим обобщенным вектором. 
или, эквивалентно,

$$
\left\langle F^{\sharp} A_{1} \mid \pi^{\sharp}\left(\mathcal{E}_{u}\right) F^{\sharp} A_{2}\right\rangle^{\sharp}=s^{\sharp}\left(A_{1}^{*} \mathcal{E}_{u} A_{2}\right), \quad A_{1}, A_{2} \in \mathcal{J}^{\sharp}, \quad u \in \chi .
$$

Соответственно, представление $A \rightarrow \pi^{\sharp}(A)$ алгебры $\mathcal{A}^{\sharp}(\chi)$ получается заменой в $(131) \pi^{\sharp}(A)$ вместо $\pi^{\sharp}\left(\mathcal{E}_{u}\right)$ и $A$ вместо $\mathcal{E}_{u}$. Построенное таким образом представление $\pi^{\sharp}$ алгебры $\mathcal{A}^{\sharp}(\chi)$ полной системы мы назовем представлением, индуцированным представлением $\pi_{0}^{\sharp}$ подалгебры $\mathcal{A}^{\sharp}\left(\chi_{0}\right)$.

От полной системы перейдем к исходной открытой системе. В разделе 2 с помощью формул (39) в классическом режиме и (51) в квантовом режиме переменные $\xi(62)$ открытой системы вкладываются в алгебру полной системы: $\xi=Y^{\sharp}(0) u$, где $u=x\left[\sum_{j} \varphi_{j} \otimes l_{j}\right]$. Точно так же в данном более общем случае переменные $\xi$ открытой системы реализуются операторами полной системы:

$$
e^{i \xi}:=\pi^{\sharp}\left(\mathcal{E}_{u}\right), \quad u=x\left[\sum_{j} \varphi_{j} \otimes l_{j}\right] .
$$

Эволюционная группа $\mathbb{R}$ действует в $\mathcal{H}^{\sharp}$ унитарными операторами $U^{\sharp}(\tau)$, непрерывно зависящими от $\tau$ в слабой операторной топологии:

$$
\mathrm{U}^{\sharp}(\tau) F^{\sharp}(A)=F^{\sharp}\left(\delta_{\tau} A\right), \quad A \in \mathcal{J}^{\sharp}, \quad \tau \in \mathbb{R} .
$$

C помощью стационарного обобщенного состояния можно "приготовить" нестационарные ("обычные") состояния алгебры $A^{\sharp}(\chi)$ :

$$
s_{B}^{\sharp}(A)=s^{\sharp}\left(B^{*} A B\right),
$$

где $B$ - элемент из $\mathcal{J}^{\sharp}$, нормированный условием $s^{\sharp}\left(B^{*} B\right)=1$. В $\mathcal{H}^{\sharp}$ определено унитарное представление $V^{\sharp}(g)=e^{i g \cdot \mathbf{Z}}$ калибровочной группы $G$ с оператором заряда Z:

$$
e^{i g \cdot \mathbf{Z}} F^{\sharp} A=F^{\sharp}\left(\gamma_{g} A\right), \quad A \in \mathcal{J}^{\sharp}, \quad g \in G .
$$

3.11. Свойства температурного представления. Существенную информацию о представлении $\pi^{\sharp}$ алгебры $\mathcal{A}^{\sharp}(\chi)$ можно получить из сужения $\pi^{\sharp}$ на подалгебру $\mathcal{A}^{\sharp}\left(\chi_{0}\right)$ калибровочно-инвариантных величин. Для этого мы перейдем в $z$-представление, отождествив пространство $\mathcal{H}^{\sharp}$ с прямым интегралом $\widetilde{\mathcal{H}^{\sharp}}:=\int \mathcal{H}_{z}^{\sharp} d^{n} z /(2 \pi)^{n}$ гильбертовых пространств $\mathcal{H}_{z}^{\sharp} \cong \mathcal{H}_{0}^{\sharp}$, индексируемых зарядом $z \in \mathcal{Z}$. $\widetilde{\mathcal{H}}^{\sharp}$ есть пополнение комплексного предгильбертова пространства непрерывных вектор-функций $f: z \mapsto f(z)$ от $z \in \mathcal{Z}$ с компактными носителями и со значениями в $\mathcal{H}_{0}^{\sharp}$. Скалярное произведение двух таких функций дается формулой

$$
\left\langle f_{1} \mid f_{2}\right\rangle^{\sharp}=\int\left\langle f_{1}(z) \mid f_{2}(z)\right\rangle^{\sharp} \frac{d^{n} z}{(2 \pi)^{n}} .
$$

Для установления изоморфизма между $\mathcal{H}^{\sharp}$ и $\widetilde{\mathcal{H}}^{\sharp}$ фиксируем некоторое линейное сечение $P: \mathcal{Z} \rightarrow \chi$ расслоения $\chi$ с проекцией $Z: \chi \rightarrow \mathcal{Z}$, так что $Z P z \equiv z$ при $z \in \mathcal{Z}$. Сопоставим элементу $A \in \mathcal{J}^{\sharp}$ непрерывную вектор-функцию $z \mapsto f_{A}^{\sharp}$ из $\widetilde{\mathcal{H}}^{\sharp}$ по формуле

$$
f_{A}^{\sharp}(z):=\pi_{0}^{\sharp}\left(m^{\sharp}\left(\mathcal{E}_{-P z} A\right)\right)|\mathrm{GIBBS}\rangle^{\sharp}, \quad A \in \mathcal{J}^{\sharp} .
$$


Для элементов $A, B \in \mathcal{J}^{\sharp}$ вида (111) имеет место равенство

$$
\left\langle F^{\sharp} A \mid F^{\sharp} B\right\rangle^{\sharp}=\left\langle f_{A}^{\sharp} \mid f_{B}^{\sharp}\right\rangle^{\sharp},
$$

так как обе части его приводятся к виду

$$
\int\left[\int(2 \pi)^{n} \delta(Z(u-\nu))\left\langle\left.\mathrm{GIBBS}\right|^{\sharp} \pi_{0}^{\sharp}\left(\mathcal{E}_{u}^{*} \mathcal{E}_{\nu}\right) \mid \mathrm{GIBBS}\right\rangle^{\sharp} d \bar{\rho}_{A}(u)\right] d \rho_{B}(\nu) .
$$

Следовательно, отображения $A \mapsto F^{\sharp} A$ и $A \mapsto f_{A}^{\sharp}\left(A \in \mathcal{J}^{\sharp}\right)$ имеют одинаковое нуль-пространство, и отображение $A \mapsto f_{A}^{\sharp}$ опускается до изометрического отображения векторов $F^{\sharp} A$ на плотное подмножество векторов $f_{A}^{\sharp}$ в $\widetilde{\mathcal{H}}^{\sharp}$. Так можно отождествить $\mathcal{H}^{\sharp}$ и $\widetilde{\mathcal{H}}^{\sharp}$. При этом отождествлении операторы калибровочных преобразований $V^{\sharp}(g)$ действуют как операторы умножения:

$$
\left(V^{\sharp}(g) f\right)(z)=\left(e^{i g \cdot \mathbf{Z}} f\right)(z)=e^{i g \cdot z} f(z),
$$

так что алгебра фон Неймана, порожденная оператором заряда, изоморфна алгебре функций $L^{\infty}\left(\mathcal{Z} ; d^{n} z /(2 \pi)^{n}\right)$.

Рассмотрим, как действуют операторы $E_{u}=\pi^{\mathrm{qu}}\left(\mathcal{E}_{u}\right)(u \in \chi)$ после отождествления $\mathcal{H}^{\text {qu }}$ с $\widetilde{\mathcal{H}}^{\text {qu }}$. Взяв вектор $f$ в виде $f_{A}^{\text {qu }}$, нетрудно убедиться, что при $u \in \chi_{0}$, $z, z_{1} \in \mathcal{Z}, B=\mathcal{E}_{u} \mathcal{E}_{P z_{1}} A \in \mathcal{J}^{\text {qu }}$ справедливо

$$
\begin{array}{r}
\left(\pi^{\mathrm{qu}}\left(\mathcal{E}_{u} \mathcal{E}_{P z_{1}}\right) f_{A}^{\mathrm{qu}}\right)(z)=f_{B}^{\mathrm{qu}}(z)=\pi_{0}^{\mathrm{qu}}\left(m^{\mathrm{qu}}\left(\mathcal{E}_{-P z} \mathcal{E}_{u} \mathcal{E}_{P z_{1}} A\right)\right)|\mathrm{GIBBS}\rangle^{\sharp}= \\
=e^{i \hbar \sigma(u, P z)} e^{(i \hbar / 2) \sigma\left(P z_{1}, P z\right)} \pi_{0}\left(\mathcal{E}_{u}\right) \pi_{0}^{\mathrm{qu}}\left(m^{\mathrm{qu}}\left(\mathcal{E}_{-P\left(z-z_{1}\right)} A\right)\right)|\mathrm{GIBBS}\rangle^{\sharp},
\end{array}
$$

так что

$$
\left(\pi^{\mathrm{qu}}\left(\mathcal{E}_{u} \mathcal{E}_{P z_{1}}\right) f_{A}^{\mathrm{qu}}\right)(z)=e^{i \hbar \sigma(u, P z)} e^{(i \hbar / 2) \sigma\left(P z_{1}, P z\right)} \pi_{0}^{\mathrm{qu}}\left(\mathcal{E}_{u}\right) f_{A}^{\mathrm{qu}}\left(z-z_{1}\right) .
$$

Такая же формула имеет место для всех $f \in \widetilde{\mathcal{H}}^{\text {qu }}, u \in \chi_{0}, z, z_{1} \in \mathcal{Z}$ :

$$
\left(\pi^{\mathrm{qu}}\left(\mathcal{E}_{u} \mathcal{E}_{P z_{1}}\right) f\right)(z)=e^{i \hbar \sigma(u, P z)} e^{(i \hbar / 2) \sigma\left(P z_{1}, P z\right)} \pi_{0}^{\mathrm{qu}}\left(\mathcal{E}_{u}\right) f\left(z-z_{1}\right) .
$$

В классическом режиме имеет место аналогичная формула:

$$
\left(\pi^{\mathrm{cl}}\left(\mathcal{E}_{u} \mathcal{E}_{P z_{1}}\right) f\right)(z)=\pi^{\mathrm{cl}}\left(\mathcal{E}_{u}\right) f\left(z-z_{1}\right) .
$$

Из (138) при $z_{1}=0$ получаем

$$
\left(\pi^{\mathrm{qu}}\left(\mathcal{E}_{u}\right) f\right)(z)=\pi_{0}^{\mathrm{qu}}\left(e^{i \hbar \sigma(u, P z)} \mathcal{E}_{u}\right) f(z), \quad u \in \chi_{0}, \quad z \in \mathcal{Z} .
$$

Эта формула показывает, что операторы $\pi^{\mathrm{qu}}\left(\mathcal{E}_{u}\right)$ при $u \in \chi_{0}$ приводятся в прямом интеграле для $\widetilde{\mathcal{H}}^{\text {qu }}$ семейством операторов $\pi_{z}^{\text {qu }}\left(\mathcal{E}_{u}\right)$, которые получаются из гиббсовского представления $\pi_{0}^{\mathrm{qu}}\left(\mathcal{E}_{u}\right)$ линейными неоднородными каноническими преобразованиями

$$
Y_{0}(0) u \rightarrow Y_{0}(0) u+\hbar \sigma(u, P z), \quad u \in \chi_{0}, \quad z \in \mathcal{Z} .
$$

Поэтому представления $\pi_{z}$ алгебры $\mathcal{A}^{\mathrm{qu}}\left(\chi_{0}\right)$ можно назвать смещенными гиббсовскими представлениями. Им соответствуют автоморфизмы $\alpha_{z}$ подалгебры $\mathcal{A}^{\text {qu }}\left(\chi_{0}\right)$, которые определяются на элементах $\mathcal{E}_{u}$ посредством

$$
\alpha_{z}\left(\mathcal{E}_{u}\right)=e^{i \hbar \sigma(u, P z)} \mathcal{E}_{u}=e^{2 i(u \mid b(z))^{\mathrm{qu}} / \beta} \mathcal{E}_{u}, \quad u \in \chi_{0}, \quad z \in \mathcal{Z}
$$


здесь и далее

$$
b(z)=J \omega D P z \in H_{0}^{\mathrm{qu}} .
$$

ПреДЛОжЕниЕ 1. При температуре $T>0$ представления $\pi_{z}^{\mathrm{qu}}$ алгебры $\mathcal{A}^{\mathrm{qu}}\left(\chi_{0}\right)$ унитарно эквивалентны представлению $\pi_{0}^{\mathrm{qu}}$.

ДокАзАтЕльство. В лемме 1 (см. приложение) показано, что в пространстве $\mathcal{H}_{0}^{\text {qu }}$ гиббсовского представления алгебры $\mathcal{A}^{\mathrm{qu}}\left(\chi_{0}\right)$ действует представление $\mathcal{E}_{\nu} \rightarrow$ $r\left(\mathcal{E}_{\nu}\right)$ системы KKC над $\chi_{0}$, непрерывно зависящее (в слабой операторной топологии) от $\nu \in \chi_{0}$ по норме $\|\nu\|^{\text {qu }}$ такое, что

$$
r\left(\mathcal{E}_{\nu}\right) \pi_{0}^{\mathrm{qu}}\left(\mathcal{E}_{u}\right) r\left(\mathcal{E}_{\nu}\right)^{-1}=e^{2 i(u \mid \nu)^{\mathrm{qu}} / \beta} \pi_{0}^{\mathrm{qu}}\left(\mathcal{E}_{u}\right), \quad u \in \chi_{0}
$$

Сравнивая это выражение с формулой (142), мы видим, что автоморфизм $\alpha_{z}$ реализуем унитарным оператором $W_{z}:=r\left(\mathcal{E}_{\nu}\right)$ при $\nu=b(z)$. Это доказывает предложение 1.

Представляет интерес вопрос, является ли оператор заряда динамической переменной в квантовом случае, когда $\mathbf{Z}$ интерпретируется как генератор калибровочных преобразований. Он действует как оператор умножения на $z:(g \cdot \mathbf{Z} f)(z)=(g \cdot z) f(z)$. Оператор заряда может считаться динамической переменной только при условии, что он присоединен к алгебре фон Неймана $\pi^{\mathrm{qu}}\left(\mathcal{A}^{\mathrm{qu}}(\chi)\right)^{\prime \prime}$, порожденной основными динамическими переменными $Y^{\mathrm{qu}}(t) u$ (или $\xi$, см. (62)) в представлении $\pi^{\mathrm{qu}}$. Однако это условие не выполнено в рассматриваемом случае конечной температуры.

ПрЕДЛОЖЕНИЕ 2. При температуре $T>0$ компоненты $g \cdot \mathbf{Z}$ оператора заряда nри $g \in G, g \neq 0$ не присоединены к алгебре фон Неймана $\pi^{\mathrm{qu}}\left(\mathcal{A}^{\mathrm{qu}}(\chi)\right)^{\prime \prime}$, и потому $\mathbf{Z}$ не являтся динамической переменной.

ДокАзАтельство. Определим унитарный оператор $W$ в $\widetilde{\mathcal{H}}^{\text {qu }}$, полагая $(W f)(z)$ $:=W_{z} f(z)$, где $f \in \widetilde{\mathcal{H}}^{\text {qu }}, z \in \mathcal{Z}$, а $W_{z}$ - оператор, определенный в доказательстве предложения 1. Формула $\tilde{\pi}^{\mathrm{qu}}(A):=W \pi^{\mathrm{qu}}(A) W^{-1}$ реализует в $\widetilde{\mathcal{H}}^{\text {qu }}$ представление $\tilde{\pi}^{\text {qu }}$ алгебры $\mathcal{A}^{\text {qu }}(\chi)$, унитарно-эквивалентное $\pi^{\text {qu}}$. Далее удобно рассматривать $\widetilde{\mathcal{H}}^{\text {qu }}$ как тензорное произведение $\widetilde{\mathcal{H}}^{\text {qu }}=\widetilde{\mathcal{H}}_{0}^{\text {qu }} \otimes L^{2}\left(\mathcal{Z} ; d^{n} z /(2 \pi)^{n}\right)$. При этом операторы калибровочных преобразований остаются операторами умножения: $V(g)=1 \otimes e^{i g \cdot \hat{z}}$, где $(\hat{z} h)(z):=z \cdot h(z)$. Операторы представления $\tilde{\pi}^{q u}$ принимают вид (здесь учтена формула $W_{z}:=r\left(\mathcal{E}_{\nu}\right)$ при $\left.\nu=b(z)\right)$ :

$$
\begin{aligned}
\tilde{\pi}^{\mathrm{qu}}\left(\mathcal{E}_{u}\right) & =\pi_{0}^{\mathrm{qu}}\left(\mathcal{E}_{u}\right) \otimes 1, \quad u \in \chi_{0}, \\
\tilde{\pi}^{\mathrm{qu}}\left(\mathcal{E}_{P z_{1}}\right) & =r\left(\mathcal{E}_{b\left(z_{1}\right)}\right) \otimes e^{-(i \hbar / 2) \sigma\left(P \hat{z}, P z_{1}\right)} e^{(i \hbar / 2) \sigma\left(b(\hat{z}), b\left(z_{1}\right)\right)} e^{i \hat{g} \cdot z_{1}},
\end{aligned}
$$

где $z_{1} \in \mathcal{Z}, \hat{g}=i \partial_{z}$. Нетрудно убедиться, что операторы

$$
R\left(z_{1}\right):=1 \otimes e^{(i \hbar / 2) \sigma\left(P \hat{z}, P z_{1}\right)} e^{-(i \hbar / 2) \sigma\left(b(\hat{z}), b\left(z_{1}\right)\right)} e^{i \hat{g} \cdot z_{1}}
$$

коммутируют с операторами $\tilde{\pi}^{\mathrm{qu}}\left(\mathcal{E}_{u}\right)$ и $\tilde{\pi}^{\mathrm{qu}}\left(\mathcal{E}_{P z_{2}}\right)\left(u \in \chi_{0}, z_{2} \in \mathcal{Z}\right)$. Предположим, что $V(g) \in \tilde{\pi}^{\text {qu }}\left(\mathcal{A}^{\text {qu}}(\chi)\right)^{\prime \prime}$. Тогда $V(g)$ коммутирует с операторами $R\left(z_{1}\right)$ и, следовательно,

$$
e^{i \hat{g} \cdot z_{1}} e^{i g \cdot \hat{z}} e^{-i \hat{g} \cdot z_{1}}=e^{i g \cdot \hat{z}}
$$

откуда получаем $e^{i g \cdot\left(\hat{z}+z_{1}\right)}=e^{i g \cdot \hat{z}}$ при всех $z_{1} \in \mathcal{Z}$. Это означает, что $g=0$. Таким образом, оператор $g \cdot \mathbf{Z}$ присоединен к алгебре фон Неймана $\pi^{\mathrm{qu}}\left(\mathcal{A}^{\mathrm{qu}}(\chi)\right)^{\prime \prime}$ только при $g=0$. Это доказывает предложение 2. 
Заметим, что в классическом режиме справедливы утверждения, аналогичные предложениям 1,2 . То, что оператор заряда не присоединен к $\pi^{\mathrm{cl}}\left(\mathcal{A}^{\mathrm{cl}}(\chi)\right)^{\prime \prime}$, очевидно из того, что он не коммутирует с $\pi^{\mathrm{cl}}\left(\mathcal{E}_{P z_{1}}\right)$ при $z_{1} \neq 0$. Сравним квантовые температурные представления с вакуумным представлением (соответствующим нулевой температуре). В работе [24] (см. также [23], §11.1) было показано, что при условиях, сопоставимых с условиями 1-4 (см. п. 3.4-3.6), для квантового вакуумного представления системы (названной там конечно вырожденной бозонной системой) вместо справедливости утверждений типа предложений 1, 2 имеет место совершенно противоположная ситуация ${ }^{12)}$. Это означает, что по своим свойствам квантовое представление $\pi^{\text {qu }}$ при конечной температуре ближе к классическому представлению $\pi^{\mathrm{cl}}$, чем к квантовому вакуумному представлению.

\section{Смещенные квазисвободные состояния алгебры ККС}

В обозначениях п. 3.9 рассмотрим квазисвободное состояние (с нулевым средним) $s_{0}^{\text {qu }}$ системы $\mathrm{KKC}\left\{\mathcal{E}_{u}\right\}$ над вещественным предгильбертовым пространством $\chi_{0}$ со скалярным произведением $(u \mid \nu)^{\mathrm{qu}}\left(u, \nu \in \chi_{0}\right)$. Обозначим через $H_{0}^{\text {qu }}$ пополнение $\chi_{0}$ по норме $\|u\|^{\text {qu}}$. Характеристический функционал состояния $s_{0}^{\text {qu }}$ имеет вид $s_{0}^{\mathrm{qu}}\left(\mathcal{E}_{u}\right)=e^{-\left(\|u\|^{\mathrm{qu}}\right)^{2} / 2 \beta}$, где $\beta=1 / k T$. Унитарные элементы $\mathcal{E}_{u}$ Удовлетворяют ККС в форме Вейля:

$$
\mathcal{E}_{u} \mathcal{E}_{\nu}=e^{(i \hbar / 2) \sigma(u, \nu)} \cdot \mathcal{E}_{u+\nu}, \quad u, \nu \in H_{0}^{\mathrm{qu}}
$$

Здесь $\sigma(u, \nu)$ - симплектическая форма над $H_{0}^{\text {qu }}$ вида $(123)$, где $\omega$ - симметрический оператор в $H_{0}^{\text {qu }}, 0<\omega<1, J$ - оператор комплексной структуры в $H_{0}^{\text {qu }}$, коммутирующий с $J$, т. е. $J^{*}=-J, J^{2}=-1, J \omega=\omega J$. Далее для краткости вместо $H_{0}^{\mathrm{qu}},\|u\|^{\mathrm{qu}},(u \mid \nu)^{\mathrm{qu}}, s_{0}^{\mathrm{qu}}, \pi_{0}^{\mathrm{qu}},|\mathrm{GIBBS}\rangle^{\mathrm{qu}}$ мы пишем просто $H_{0},\|u\|,(u \mid \nu), s_{0}, \pi_{0}$, $|\mathrm{GIBBS}\rangle$. Процедура ГНС сопоставляет квазисвободному состоянию $s_{0}$ представление $\pi_{0}: \mathcal{E}_{u} \mapsto \pi_{0}\left(\mathcal{E}_{u}\right)$ системы KKC в комплексном гильбертовом пространстве $\mathcal{H}_{0}$ с циклическим вектором $|\mathrm{GIBBS}\rangle$.

Известно [18]-[20], что построенную с помощью $s_{0}$ конкретную систему $\mathrm{KKC}$ $\pi_{0}\left(\mathcal{E}_{u}\right)$ можно рассматривать как подсистему некоторой фоковской системы. С этой целью введем вещественное симплектическое пространство $H^{\mathrm{F}}:=F \oplus F$ с симплектической формой $\sigma^{\mathrm{F}}\left(u \oplus u^{\prime}, \nu \oplus \nu^{\prime}\right):=\sigma(u, \nu)-\sigma\left(u^{\prime}, \nu^{\prime}\right)$, где $u, u^{\prime}, \nu, \nu^{\prime} \in F$, а $F-$ общая область определения операторов $\omega^{-n}, n=1,2, \ldots$, в $H_{0}(F-$ плотный линеал в $\left.H_{0}\right) . H^{\mathrm{F}}$ есть вещественное предгильбертово пространство с квадратом нормы

$$
\left\|u \oplus u^{\prime}\right\|^{2}:=\|u\|^{2}-2\left(u \mid \sqrt{1-\omega^{2}} u^{\prime}\right)+\left\|u^{\prime}\right\|^{2}
$$

и с соответствующим скалярным произведением $\left(u \oplus u^{\prime} \mid \nu \oplus \nu^{\prime}\right)$. Очевидно, оператор вложения $u \mapsto u \oplus 0 \in H^{\mathrm{F}}, u \in F$, сохраняет скалярное произведение и симплектическую форму. Нетрудно убедиться, что формула

$$
J^{\mathrm{F}} u \oplus u^{\prime}=\omega^{-1} J\left(u-\sqrt{1-\omega^{2}} u^{\prime}\right) \oplus \omega^{-1} J\left(\sqrt{1-\omega^{2}} u-u^{\prime}\right)
$$

12) В п.4.1, 4.3 работы [14] были сделаны утверждения о представлении для квантового броуновского движения (при $T>0$ ) со ссылкой на аналогию с квантовым вакуумным представлением. Предложения 1, 2 настоящей работы должны заменить эти утверждения, поскольку указанная аналогия неправомерна. 
определяет на $H^{\mathrm{F}}$ изометрический оператор, квадрат которого равен -1 (так что $J^{\mathrm{F}}$ - оператор комплексной структуры). При этом

$$
\sigma^{\mathrm{F}}\left(u \oplus u^{\prime}, \nu \oplus \nu^{\prime}\right)=\frac{2}{\beta \hbar}\left(u \oplus u^{\prime} \mid J^{\mathrm{F}} \nu \oplus \nu^{\prime}\right)
$$

Рассмотрим систему $\mathrm{KKC} \mathrm{над} H^{\mathrm{F}}$ с унитарными элементами $\mathcal{E}_{u \oplus u^{\prime}}$, удовлетворяющими соотношениям

$$
\mathcal{E}_{u \oplus u^{\prime}} \mathcal{E}_{\nu \oplus \nu^{\prime}}=e^{(i \hbar / 2) \sigma^{\mathrm{F}}\left(u \oplus u^{\prime}, \nu \oplus \nu^{\prime}\right)} \mathcal{E}_{(u+\nu) \oplus\left(u^{\prime}+\nu^{\prime}\right)}
$$

Как известно (из метода вторичного квантования), существует фоковское представление $\mathcal{E}_{u \oplus u^{\prime}} \mapsto E_{u \oplus u^{\prime}}$ этой системы в комплексном гильбертовом пространстве $\mathcal{H}^{\mathrm{F}}$ с циклическим фоковским вакуумным вектором $|\mathrm{FOCK}\rangle$. Характеристический функционал этого состояния есть $\left\langle\mathrm{FOCK}\left|E_{u \oplus u^{\prime}}\right| \mathrm{FOCK}\right\rangle=e^{-\left\|u \oplus u^{\prime}\right\|^{2} / 2 \beta}$, откуда следует непрерывность $E_{u \oplus u^{\prime}}$ (в слабой операторной топологии для $E_{u \oplus u^{\prime}}$ и по норме $\left.\left\|u \oplus u^{\prime}\right\|\right)$. При этом подсистема $E_{u \oplus 0}$ имеет тот же характеристический функционал, что и $\pi_{0}\left(\mathcal{E}_{u}\right)$ в состоянии с циклическим вектором $\mid$ GIBBS $\rangle$. Нетрудно убедиться, что линеал $(F \oplus 0)+J^{\mathrm{F}}(F \oplus 0)$ плотен в $H^{\mathrm{F}}$. Из этого можно сделать вывод, что вектор $|\mathrm{FOCK}\rangle$ является циклическим для подсистемы $E_{u \oplus 0}$ при $u \in F$ (аргументация приведена в доказательстве теоремы 4.1 в [21]). Тем самым исходную систему $\pi_{0}\left(\mathcal{E}_{u}\right)$ в $\mathcal{H}_{0}$ с циклическим вектором $|\mathrm{GIBBS}\rangle$ можно отождествить с подсистемой $E_{u \oplus 0}$ при $u \in F$ в $\mathcal{H}^{\mathrm{F}}$ с циклическим вектором $|\mathrm{FOCK}\rangle$. Это позволяет доказать следующую лемму.

Лемма 1. В пространстве $H^{\mathrm{F}}$ действует представление $r: \mathcal{E}_{\nu} \mapsto r\left(\mathcal{E}_{\nu}\right)$ KКC над $\chi_{0}$ со свойствами

$$
\begin{aligned}
r\left(\mathcal{E}_{\nu}\right) \pi_{0}\left(\mathcal{E}_{u}\right) r\left(\mathcal{E}_{\nu}\right)^{-1} & =e^{2 i(u \mid \nu) / \beta} \pi_{0}\left(\mathcal{E}_{u}\right), \quad u, \nu \in \chi_{0}, \\
\left\langle\operatorname{GIBBS}\left|r\left(\mathcal{E}_{\nu}\right)\right| \mathrm{GIBBS}\right\rangle & =e^{-\|\nu\|^{2} / 2 \beta} .
\end{aligned}
$$

ДокАЗАТЕЛЬСтво. Отождествим систему $\pi_{0}\left(\mathcal{E}_{u}\right)$ при $u \in F$ с подсистемой $E_{u \oplus 0}$ при $u \in F$ в $\mathcal{H}^{\mathrm{F}}$ и положим $r\left(\mathcal{E}_{\nu}\right)=E_{w \oplus w^{\prime}}$ при $w \oplus w^{\prime}=J^{\mathrm{F}}(\nu \oplus 0)$. Нетрудно убедиться, что при этом выполняются соотношения (П.2) при $u, \nu \in F$, а по непрерывности они переносятся на $H_{0}$ или на $\chi_{0}$.

Из леммы непосредственно вытекает следующий критерий унитарной реализуемости неоднородного линейного канонического преобразования системы KKC, которое в экспоненциальной форме Вейля записывается как

$$
\mathcal{E}_{u} \mapsto e^{i q(u) / \beta} \mathcal{E}_{u}, \quad u \in \chi_{0},
$$

где $u \mapsto q(u)$ - линейная форма на $\chi_{0}$. (В одну сторону (только тогда) этот критерий вполне очевиден.)

СлеДСтвиЕ 7. В представлении $\pi_{0}: \mathcal{E}_{u} \mapsto \pi_{0}\left(\mathcal{E}_{u}\right)$ KKC над $\chi_{0}, u \in \chi_{0}$, каноническое преобразование (П.3) унитарно реализуемо тогда и только тогда, когда линейная форма $q(u)$ непрерьвна по норме $\|u\|$, m.е. (П.3) имеет вид

$$
\mathcal{E}_{u} \mapsto e^{2 i\left(u \mid \nu_{0}\right) / \beta} \mathcal{E}_{u} \quad \text { при некотором } \quad \nu_{0} \in H_{0} .
$$


Если унитарный оператор U, реализующий каноническое преобразование (П.3), принадлежит алгебре фон Неймана $\left\{\pi_{0}\left(\mathcal{E}_{u}\right)\right\}^{\prime \prime}$, то это налагает дополнительное ограничение на $q(u)$ в (П.3), а именно:

$$
q(u)=\beta \hbar \sigma\left(u, w_{0}\right) \quad \text { при некотором } \quad w_{0} \in H_{0} .
$$

Для доказательства воспользуемся тем же, что и в доказательстве леммы 1 , отождествлением $\pi_{0}\left(\mathcal{E}_{u}\right)$ с $E_{u \oplus 0}$ при $u \in F$. Имеем

$$
r\left(\mathcal{E}_{\nu}\right)=E_{w \oplus w^{\prime}}=E_{w \oplus 0} E_{0 \oplus w^{\prime}}=\pi_{0}\left(\mathcal{E}_{w}\right) E_{0 \oplus w^{\prime}},
$$

где $\nu, w, w^{\prime} \in F, w \oplus w^{\prime}=J^{\mathrm{F}} \nu \oplus 0, w=J \omega^{-1} \nu$. Учтем, что оператор U перестановочен с $E_{0 \oplus w^{\prime}}$, получим

$$
\mathrm{U} r\left(\mathcal{E}_{\nu}\right) \mathrm{U}^{-1}=\left(\mathrm{U} \pi_{0}\left(\mathcal{E}_{w}\right) \mathrm{U}^{-1}\right) E_{0 \oplus w^{\prime}}=e^{i q(w) / \beta} \pi_{0}\left(\mathcal{E}_{w}\right) E_{0 \oplus w^{\prime}}=e^{i q(w) / \beta} r\left(\mathcal{E}_{\nu}\right) .
$$

Так как левая часть этих соотношений, очевидно, непрерывна по норме $\|\nu\|$, то $q(w)$ есть линейный непрерывный функционал от $\nu$ в топологии нормы $\|\nu\|$, поэтому $q(w)=2\left(\nu \mid w_{0}\right)$ при некотором $w_{0} \in H_{0}$. Таким образом, $q(w)=-2\left(J \omega w \mid w_{0}\right)=$ $2\left(w \mid J \omega w_{0}\right)=\beta \hbar \sigma\left(w, w_{0}\right)$ при $w \in F$, что доказывает (П.4).

Отсюда получается еще один критерий (который, как и предыдущий, в одну сторону очевиден).

СлЕДСТВИЕ 8. В представлении $\pi_{0}: \mathcal{E}_{u} \mapsto \pi_{0}\left(\mathcal{E}_{u}\right) K K C$ над $\chi_{0}\left(\right.$ әде $\left.u \in \chi_{0}\right)$ каноническое преобразование (П.3) реализуемо унитарным оператором из алгебры фон Неймана $\left\{\pi_{0}\left(\mathcal{E}_{u}\right)\right\}^{\prime \prime}$ в точности тогда, когда (П.3) имеет вид

$$
\mathcal{E}_{u} \mapsto e^{i \hbar \sigma\left(u, w_{0}\right)} \mathcal{E}_{u} \quad \text { при некотором } \quad w_{0} \in H_{0} .
$$

Заметим, что при каноническом преобразовании (П.3) состояние $s_{0}$ переходит в состояние с характеристическим функционалом $e^{i q(u) / \beta} s_{0}\left(\mathcal{E}_{u}\right)$. Оно называется смещенным квазисвободным состоянием алгебры KKC, так как элементы $Y_{0}(u)$, входящие в соотношение $\pi_{0}\left(\mathcal{E}_{u}\right)=e^{i Y_{0}(u)}$, приобретают в этом состоянии среднее $q(u) / \beta$.

Благодарности. Автор благодарен А. Д. Суханову за плодотворные дискуссии.

\section{Список литературы}

[1] G. W. Ford, M. Kac, P. Mazur, J. Math. Phys., 6:4 (1965), 504-515.

[2] P. Ullersma, Physica, 32:1 (1966), 27-55; 56-73; 74-89; 90-96.

[3] J. T. Lewis, J. V. Pulè, "Dynamical theory of Brownian motion", International Symposium on Mathematical Problems in Theoretical Physics (Kyoto, Japan, January 23-29, 1975), Lecture Notes in Physics, 39, ed. H. Araki, Springer, Berlin, 1975, 516-519.

[4] Д. Т. Льюис, Г. Маасен, "Гамильтоновы модели классических и квантовых случайных процессов", Квантовые случайные прочессы и открытые системъ, Мир, М., 1988, $53-91$.

[5] R. Benguria, M. Kac, Phys. Rev. Lett., 46:1 (1981), 1-4.

[6] J. T. Lewis, L. C. Thomas, "How to make a heat bath", Functional Integration and Its Applications, Proceedings of the International Conference (Cumberland Lodge, London, April 1974), ed. A. M. Arthurs, Clarendon Press, Oxford, 1975, 93-123. 
[7] Th. M. Nieuwenhuizen, A.E. Allahverdyan, Phys. Rev. E, 66:3 (2002), 036102, 52 pp.

[8] G. W. Ford, J. T. Lewis, R. F. O'Connell, Phys. Rev. A, 37:11 (1988), 4419-4428.

[9] H. Dekker, Phys. Rep., 80:1 (1981), 1-110.

[10] R. F. Feynman, F. L. Vernon, Ann. Phys., 24:1 (1963), 118-173.

[11] A. O. Caldeira, A. J. Leggett, Ann. Phys., 149:2 (1983), 374-456.

[12] H. Grabert,P. Schramm, G.-L. Ingold, Phys. Rep., 168:3 (1988), 115-207.

[13] И. П. Корнфельд, Я. Г. Синай, С. В. Фомин, Эргодическая теория, Наука, М., 1980.

[14] А. И. Оксак, А. Д. Суханов, ТМФ, 136:1 (2003), 115-147.

[15] Ю. Г. Рудой, А. Д. Суханов, УФН, 170:12 (2000), 1265-1296.

[16] Л. Д. Ландау, Е. М. Лифшиц, Теоретическая физика, т. 5: Статистическая физика, Наука, М., 1976.

[17] А. Мессиа, Квантовая механика, т. 1, Наука, М., 1978.

[18] J. Manuceau, A. Verbeure, Commun. Math. Phys., 9:4 (1968), 293-302.

[19] J. Manuceau, F. Rocca, D. Testard, Commun. Math. Phys., 12:1 (1969), 43-57.

[20] А. С. Холево, ТМФ, 6:1 (1971), 3-20; 6:2, 145-150.

[21] H. Araki, E. J. Woods, J. Math. Phys., 4:5 (1963), 637-662.

[22] Ж. Диксмье, $C^{*}$-алгебры и их представления, Наука, М., 1974.

[23] Н. Н. Боголюбов, А. А. Логунов, А. И. Оксак, И. Т. Тодоров, Общие принципь квантовой теории поля, Физматгиз, М., 2006.

[24] А. И. Оксак, ТМФ, 48:3 (1981), 297-318.

[25] А. Барут, Р. Рончка, Теория представлений групп и ее приложения, Т. 2, Мир, М., 1980.

[26] Т. Я. Азизов, И. С. Иохвидов, Основы теории линейных операторов в пространствах с индефинитной метрикой, Наука, М., 1986.

[27] И. Сигал, Математические проблемы релятивистской физики, Мир, М., 1968.

[28] D. Kastler, Commun. Math. Phys., 1:1 (1965), 14-48.

[29] M. Nakamura, T. Turumaru, Tôhoku Math. J., 6:2-3 (1954), 182-188.

[30] П. А. М. Дирак, Принципы квантовой механики, Наука, М., 1979.

Поступила в редакцию 4.04.2012, после доработки 12.07.2012 